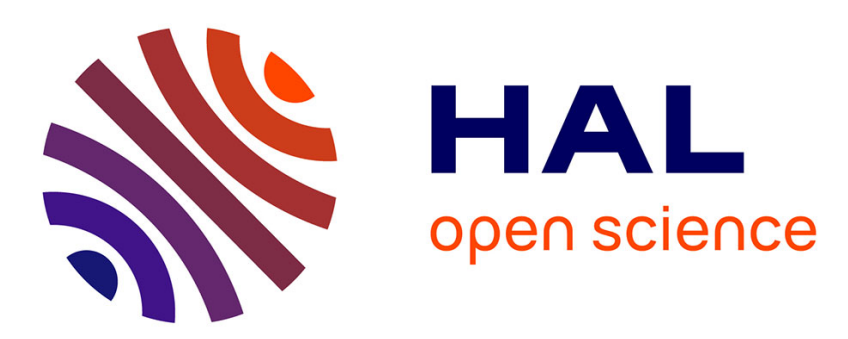

\title{
Energy Conserving Explicit Local Time-Stepping for Second-Order Wave Equations
}

\author{
Julien Diaz, Marcus J. Grote
}

\section{To cite this version:}

Julien Diaz, Marcus J. Grote. Energy Conserving Explicit Local Time-Stepping for SecondOrder Wave Equations. SIAM Journal on Scientific Computing, 2009, 31 (3), pp.1985-2014. 10.1137/070709414 . inria-00409233

\section{HAL Id: inria-00409233 \\ https://hal.inria.fr/inria-00409233}

Submitted on 6 Aug 2009

HAL is a multi-disciplinary open access archive for the deposit and dissemination of scientific research documents, whether they are published or not. The documents may come from teaching and research institutions in France or abroad, or from public or private research centers.
L'archive ouverte pluridisciplinaire HAL, est destinée au dépôt et à la diffusion de documents scientifiques de niveau recherche, publiés ou non, émanant des établissements d'enseignement et de recherche français ou étrangers, des laboratoires publics ou privés. 


\title{
ENERGY CONSERVING EXPLICIT LOCAL TIME STEPPING FOR SECOND-ORDER WAVE EQUATIONS*
}

\author{
JULIEN DIAZ ${ }^{\dagger}$ AND MARCUS J. GROTE ${ }^{\ddagger}$
}

\begin{abstract}
Locally refined meshes impose severe stability constraints on explicit time-stepping methods for the numerical simulation of time dependent wave phenomena. To overcome that stability restriction, local time-stepping methods are developed, which allow arbitrarily small time steps precisely where small elements in the mesh are located. When combined with a symmetric finite element discretization in space with an essentially diagonal mass matrix, the resulting discrete numerical scheme is explicit, is inherently parallel, and exactly conserves a discrete energy. Starting from the standard second-order "leap-frog" scheme, time-stepping methods of arbitrary order of accuracy are derived. Numerical experiments illustrate the efficiency and usefulness of these methods and validate the theory.
\end{abstract}

Key words. second-order hyperbolic problems, explicit methods, time reversible methods, energy conservation, discontinuous Galerkin methods, finite element methods, mass lumping, wave equation, acoustic waves, electromagnetic waves

AMS subject classification. 65 N30

DOI. $10.1137 / 070709414$

1. Introduction. The efficient and accurate numerical solution of the wave equation is of fundamental importance for the simulation of time dependent acoustic, electromagnetic, or elastic wave phenomena. Finite difference methods are commonly used for the simulation of time dependent waves because of their simplicity and their efficiency on structured Cartesian meshes [27, 28, 34]. However, in the presence of complex geometry or small geometric features that require locally refined meshes, their usefulness is somewhat limited. In contrast, finite element methods (FEMs) easily handle locally refined unstructured meshes; moreover, their extension to high order is straightforward, even in the presence of curved boundaries or material interfaces.

The finite element Galerkin discretization of second-order hyperbolic problems typically leads to a second-order system of ordinary differential equations. Even if explicit time stepping is employed, the mass matrix arising from the spatial discretization by standard continuous finite elements must be inverted at each time step - a major drawback in terms of efficiency. To overcome that problem, various "mass lumping" techniques have been proposed, which effectively replace the mass matrix by a diagonal approximation. While straightforward for piecewise linear elements $[8,32]$, mass lumping techniques require particular quadrature or cubature rules at higher order to preserve the accuracy and guarantee numerical stability [14, 22].

Alternatively, discontinuous Galerkin (DG) methods offer even greater flexibility for local mesh refinement by accommodating nonconforming grids and hanging nodes.

*Received by the editors November 27, 2007; accepted for publication (in revised form) December 11, 2008; published electronically March 27, 2009. This work was supported by the Swiss National Science Foundation.

http://www.siam.org/journals/sisc/31-3/70941.html

${ }^{\dagger}$ Department of Mathematics, University of Basel, Rheinsprung 21, 4051 Basel, Switzerland and INRIA Research Center Bordeaux-Sud Ouest, Team-project Magique 3D and Laboratoire de Mathématiques Appliquées, UMR CNRS 5142. Université de Pau et des Pays de l'Adour - Bt. IPRA, BP 1155, 64013 PAU Cedex (Julien.Diaz@inra.fr).

${ }^{\ddagger}$ Department of Mathematics, University of Basel, Rheinsprung 21, 4051 Basel, Switzerland (Marcus.Grote@unibas.ch). 
Based on discontinuous finite element spaces, DG-FEMs weakly enforce continuity by adding suitable bilinear forms, so-called numerical fluxes, to standard variational formulations - see $[9,10,11]$ for further details and recent reviews. Because individual elements decouple, the mass matrix arising from a spatial DG discretization is blockdiagonal, with block size equal to the number of degrees of freedom per element; it can, therefore, be inverted at very low computational cost. In fact, for a judicious choice of (locally orthogonal) shape functions, the mass matrix is diagonal. Thus, when combined with explicit time integration, the resulting time-marching scheme will be truly explicit.

Recently, Grote, Schneebeli, and Schötzau [23] proposed the symmetric interior penalty (IP) DG method for the second-order wave equation and, in particular, derived optimal a priori error bounds in the energy norm and the $L^{2}$-norm for the semidiscrete formulation. Optimal error estimates for the fully discrete DG method were proved in [26]. A symmetric DG formulation of the wave equation in its secondorder form offers the following advantage, which also pertains to the classical continuous Galerkin formulation. Since the stiffness matrix is positive semidefinite, the semidiscrete formulation inherently conserves (a discrete version of) the energy for all time. Moreover, when combined with a symmetric time-marching scheme, such as the standard leap-frog (or Störmer-Verlet) method, the resulting fully discrete formulation will also conserve a discrete energy. Thus, both formulations will be free of any (unnecessary) damping. The dispersive properties of the symmetric IP-DG method were analyzed by Ainsworth, Monk, and Muniz [1]. In [24, 25] the symmetric interior DG method was extended to Maxwell's equations in second-order form.

Adaptivity and mesh refinement are certainly key for the efficient numerical solution of partial differential equations. However, locally refined meshes impose severe stability constraints on explicit time-stepping schemes, where the maximal time step allowed by the CFL condition is dictated by the smallest elements in the mesh. When mesh refinement is restricted to a small region, the use of implicit methods, or a very small time step in the entire computational domain, are a very high price to pay. To overcome that stability restriction, various local time-stepping schemes were proposed, which allow smaller time steps precisely where the smallest elements in the mesh are located. In [15] Collino, Fouquet, and Joly proposed a local time-stepping method for the first-order wave equation; it was analyzed in $[16,33]$ and extended to elastodynamics [6] and Maxwell's equations [17]. Their approach, which is based on the introdution of a Lagrange multiplier, conserves a discrete energy. However, it requires the solution of a linear system on the interface between the coarse and the fine meshes. By combining a symplectic integrator with a DG discretization of Maxwell's equations in first-order form, Piperno [39] proposed an explicit local time-stepping scheme, which also conserves a discrete energy. All of these methods are second-order accurate in time. Alternatively, domain decomposition methods permit the use of different numerical methods or time steps in separate subdomains $[20,30]$.

Here, we propose local time-stepping methods for second-order wave equations of arbitrarily high order of accuracy, which are fully explicit and require no additional storage. The outline of our paper is as follows. In section 2 we recall both the standard continuous and the symmetric IP-DG finite element discretizations of the scalar second-order wave equation, which typically serves as a model problem for general second-order hyperbolic problems. Starting from the well-known "leap-frog" scheme, we then derive a second-order local time-stepping scheme in section 3 . With a symmetric finite element discretization in space with a (block-)diagonal mass matrix, the resulting fully discrete scheme not only is explicit and, thus, inherently parallel 
but also conserves (a discrete version of) the energy. We also show via numerical experiments how a small overlap between the fine and the coarse regions achieves an optimal CFL condition. Next, in section 4, we extend the second-order local timestepping schemes first to fourth order and then to arbitrarily high order of accuracy. In section 5 we present numerical experiments in one and two space dimensions which validate the theory and illustrate the usefulness of these local time-stepping schemes.

2. Finite element discretizations of the wave equation. We consider the scalar wave equation

$$
\begin{aligned}
u_{t t}-\nabla \cdot\left(c^{2} \nabla u\right) & =f & & \text { in }(0, T) \times \Omega, \\
u & =0 & & \text { on }(0, T) \times \partial \Omega, \\
\left.u\right|_{t=0} & =u_{0} & & \text { in } \Omega, \\
\left.u_{t}\right|_{t=0} & =v_{0} & & \text { in } \Omega,
\end{aligned}
$$

where $\Omega$ is a bounded domain in $\mathbb{R}^{2}$ or $\mathbb{R}^{3}$. Here, $f \in L^{2}\left(0, T ; L^{2}(\Omega)\right.$ ) is a (known) source term, while $u_{0} \in H_{0}^{1}(\Omega)$ and $v_{0} \in L^{2}(\Omega)$ are prescribed initial conditions. We consider homogeneous Dirichlet boundary conditions, for simplicity, and assume that the speed of propagation $c(x)$ is piecewise smooth and strictly positive. In the absence of forcing, the (continuous) energy

$$
E[u](t)=\frac{1}{2}\left\{\left\|u_{t}(t, .)\right\|^{2}+\|\nabla u(t, .)\|^{2}\right\}, \quad t \geq 0,
$$

is conserved for all time.

We shall now discretize in space (2.1)-(2.4) by using either standard continuous ( $H^{1}$-conforming) finite elements (with mass lumping) or a symmetric IP-DG discretization from [23], while leaving time continuous. Thus, we consider shaperegular meshes $\mathcal{T}_{h}$ that partition the domain $\Omega$ into disjoint elements $\{K\}$ such that $\bar{\Omega}=\cup_{K \in \mathcal{T}_{h}} \bar{K}$. The elements are triangles or quadrilaterals in two space dimensions, and tetrahedra or hexahedra in three dimensions, respectively. The diameter of element $K$ is denoted by $h_{K}$, and the mesh size $h$ is given by $h=\max _{K \in \mathcal{T}_{h}} h_{K}$.

2.1. Standard continuous Galerkin formulation. The standard continuous ( $H^{1}$-conforming) Galerkin formulation of the wave equation (2.1)-(2.4) starts from its weak formulation [36]: find $u:[0, T] \times H_{0}^{1}(\Omega) \rightarrow \mathbb{R}$ such that

$$
\begin{aligned}
\left(u_{t t}, v\right)+(c \nabla u, c \nabla v) & =(f, v) \quad \forall v \in H_{0}^{1}(\Omega), \quad t \in(0, T), \\
\left.u\right|_{t=0} & =u_{0}, \\
\left.u_{t}\right|_{t=0} & =v_{0} .
\end{aligned}
$$

Here, (.,.) denotes the standard $L^{2}$ inner product on $\Omega$.

For a given partition $\mathcal{T}_{h}$ of $\Omega$ and an approximation order $\ell \geq 1$, we wish to approximate the solution $u(t, \cdot)$ of $(2.6)-(2.8)$ in the finite element space

$$
V^{h}:=\left\{v \in H_{0}^{1}(\Omega):\left.v\right|_{K} \circ F_{K} \in \mathcal{S}^{\ell}(\hat{K}) \quad \forall K \in \mathcal{T}_{h}\right\},
$$

where $\mathcal{S}^{\ell}(\hat{K})$ is the space $\mathcal{P}^{\ell}(\hat{K})$ (for triangles or tetrahedra) or $\mathcal{Q}^{\ell}(\hat{K})$ (for quadrilaterals or hexahedra) and $F_{K}: \hat{K} \rightarrow K$ is one-to-one and maps the boundary of the reference element $\hat{K}$ to the boundary of $K$. Thus, we consider the following 
semidiscrete Galerkin approximation of (2.6)-(2.8): find $u^{h}:[0, T] \times V^{h} \rightarrow \mathbb{R}$ such that

$$
\begin{aligned}
\left(u_{t t}^{h}, v\right)+\left(c \nabla u^{h}, c \nabla v\right) & =(f, v) \quad \forall v \in V^{h}, \quad t \in(0, T), \\
\left.u^{h}\right|_{t=0} & =\Pi_{h} u_{0}, \\
\left.u_{t}^{h}\right|_{t=0} & =\Pi_{h} v_{0},
\end{aligned}
$$

where $\Pi_{h}$ denotes the $L^{2}$-projection onto $V^{h}$-see [4] for further details.

Let $\left(\phi_{i}\right)_{i=1 \ldots N}$ denote the standard nodal basis of $V^{h}$ defined by $\phi_{i}\left(x_{j}\right)=\delta_{i j}$, where $\left(x_{i}\right)_{i=1 \ldots N}$ are the nodes of the mesh. Next, we denote by $y(t), F(t), y_{0}$, and $\dot{y}_{0}$ the $N$-vectors

$$
y_{i}(t)=\left(u_{h}(t, .), \phi_{i}\right), \quad F_{i}(t)=\left(f(t, .), \phi_{i}\right), \quad y_{0, i}=\left(\Pi_{h} u_{0}, \phi_{i}\right), \quad \dot{y}_{0, i}=\left(\Pi_{h} v_{0}, \phi_{i}\right),
$$

respectively. Then, (2.10)-(2.12) is equivalent to the second-order system of ordinary differential equations

$$
\begin{aligned}
& M \frac{d^{2} y}{d t^{2}}+K y=F \\
& y(0)=y_{0}, \quad \frac{d y}{d t}(0)=\dot{y}_{0}
\end{aligned}
$$

where the $N \times N$ mass and stiffness matrices, $M$ and $K$, are defined by

$$
M_{i j}=\left(\phi_{i}, \phi_{j}\right), \quad K_{i j}=\left(c \nabla \phi_{i}, c \nabla \phi_{j}\right),
$$

respectively. The matrix $M$ is symmetric positive definite, whereas the matrix $K$ is symmetric and, in general, positive semidefinite only.

Since the matrix $M$ is not diagonal, it must be inverted at every time step of any explicit time integration scheme. To overcome this difficulty, various so-called mass lumping techniques have been developed, which essentially replace $M$ with a diagonal approximation by computing the integrals over each element $K$ with judicious quadrature rules. For $\mathcal{P}^{1}$-elements in two space dimensions, for instance, one uses the approximation

$$
\int_{K} f \simeq \frac{|K|}{3} \sum_{k=1}^{3} f\left(x_{K}^{k}\right),
$$

where $x_{K}^{k}$ are the vertices of triangle $K$. From the definition of the basis functions $\phi_{i}$, it then immediately follows that

$$
\int_{K} \phi_{i} \phi_{j} \simeq \frac{|K|}{3} \delta_{i j}
$$

Hence, $M$ is now diagonal while the spatial discretization remains second-order accurate $[8,32]$.

For $\mathcal{P}^{\ell}$-elements up to order $\ell=3$, mass lumping techniques are also available but more complicated [14], whereas the case of $\mathcal{Q}^{\ell}$ (or spectral)-elements for quadrilateral meshes and arbitrary $\ell$ is well-understood $[12,31,38,40]$. Here, the degrees of freedom associated with the basis functions $\left(\phi_{i}\right)$ coincide with the Gauss-Lobatto quadrature points on each element. As the integrals are also computed elementwise through Gauss-Lobatto quadrature, the resulting mass matrix is diagonal while the spatial accuracy is not affected $[4,5]$. 
2.2. Discontinuous Galerkin formulation. Here, we briefly recall the symmetric IP-DG fomulation from [23]. For simplicity, we assume in this section that the elements are triangles or parallelograms in two space dimensions and tetrahedra or parallelepipeds in three dimensions, respectively. Generally, we allow for irregular meshes with hanging nodes. An interior face of $\mathcal{T}_{h}$ is the (nonempty) interior of $\partial K^{+} \cap \partial K^{-}$, where $K^{+}$and $K^{-}$are two adjacent elements of $\mathcal{T}_{h}$. Similarly, a boundary face of $\mathcal{T}_{h}$ is the (nonempty) interior of $\partial K \cap \partial \Omega$, which consists of entire faces of $\partial K$. We denote by $\mathcal{F}_{h}^{\mathcal{I}}$ the set of all interior faces of $\mathcal{T}_{h}$ and by $\mathcal{F}_{h}^{\mathcal{B}}$ the set of all boundary faces, and let $\mathcal{F}_{h}=\mathcal{F}_{h}^{\mathcal{I}} \cup \mathcal{F}_{h}^{\mathcal{B}}$. Here, we generically refer to any element of $\mathcal{F}_{h}$ as a "face," both in two and in three dimensions.

For any piecewise smooth function $v$, we now introduce the following trace operators. Let $F \in \mathcal{F}_{h}^{\mathcal{I}}$ be an interior face shared by two neighboring elements $K^{+}$and $K^{-}$, and let $x \in F$; we write $\mathbf{n}^{ \pm}$to denote the outward unit normal vectors on the boundaries $\partial K^{ \pm}$. Denoting by $v^{ \pm}$the trace of $v$ taken from within $K^{ \pm}$, we define the jump and average of $v$ at $x \in F$ by

$$
\llbracket v \rrbracket:=v^{+} \mathbf{n}^{+}+v^{-} \mathbf{n}^{-}, \quad\left\{\{v\}:=\left(v^{+}+v^{-}\right) / 2,\right.
$$

respectively. On every boundary face $F \in \mathcal{F}_{h}^{\mathcal{B}}$, we set $\llbracket v \rrbracket:=v \mathbf{n}$ and $\{\{v\}\}:=v$. Here, $\mathbf{n}$ is the outward unit normal on $\partial \Omega$.

For a piecewise smooth vector-valued function $\mathbf{q}$, we analogously define the average across interior faces by $\left\{\{\mathbf{q}\}:=\left(\mathbf{q}^{+}+\mathbf{q}^{-}\right) / 2\right.$, and on boundary faces we set $\{\{\mathbf{q}\}:=\mathbf{q}$. The jump of a vector-valued function will not be used. For a vector-valued function $\mathbf{q}$ with continuous normal components across a face $f$, the trace identity

$$
v^{+}\left(\mathbf{n}^{+} \cdot \mathbf{q}^{+}\right)+v^{-}\left(\mathbf{n}^{-} \cdot \mathbf{q}^{-}\right)=\llbracket v \rrbracket \cdot\{\{\mathbf{q}\} \quad \text { on } f
$$

immediately follows from the above definitions.

For a given partition $\mathcal{T}_{h}$ of $\Omega$ and an approximation order $\ell \geq 1$, we wish to approximate the solution $u(t, \cdot)$ of $(2.1)-(2.4)$ in the finite element space

$$
V^{h}:=\left\{v \in L^{2}(\Omega):\left.v\right|_{K} \in \mathcal{S}^{\ell}(K) \quad \forall K \in \mathcal{T}_{h}\right\},
$$

where $\mathcal{S}^{\ell}(K)$ is the space $\mathcal{P}^{\ell}(K)$ of polynomials of total degree at most $\ell$ on $K$ if $K$ is a triangle or a tetrahedra, or the space $\mathcal{Q}^{\ell}(K)$ of polynomials of degree at most $\ell$ in each variable on $K$ if $K$ is a parallelogram or a parallelepiped. Thus, we consider the following (semidiscrete) DG approximation of (2.1)-(2.4): find $u^{h}: \bar{J} \times V^{h} \rightarrow \mathbb{R}$ such that

$$
\begin{aligned}
\left(u_{t t}^{h}, v\right)+a_{h}\left(u^{h}, v\right) & =(f, v) \quad \forall v \in V^{h}, \quad t \in J, \\
\left.u^{h}\right|_{t=0} & =\Pi_{h} u_{0}, \\
\left.u_{t}^{h}\right|_{t=0} & =\Pi_{h} v_{0} .
\end{aligned}
$$

Here, $\Pi_{h}$ denotes the $L^{2}$-projection onto $V^{h}$ and the discrete bilinear form $a_{h}$ on $V^{h} \times V^{h}$ is given by

$$
\begin{aligned}
a_{h}(u, v):= & \sum_{K \in \mathcal{T}_{h}} \int_{K} c^{2} \nabla u \cdot \nabla v d x-\sum_{F \in \mathcal{F}_{h}} \int_{F} \llbracket u \rrbracket \cdot\left\{\left\{c^{2} \nabla v\right\}\right\} d A \\
& -\sum_{F \in \mathcal{F}_{h}} \int_{F} \llbracket v \rrbracket \cdot\left\{\left\{c^{2} \nabla u\right\}\right\} d A+\sum_{F \in \mathcal{F}_{h}} \int_{F} \mathrm{a} \llbracket u \rrbracket \cdot \llbracket v \rrbracket d A .
\end{aligned}
$$


The last three terms in (2.19) correspond to jump and flux terms at element boundaries; they vanish when $u, v \in H_{0}^{1}(\Omega) \cap H^{1+\sigma}(\Omega)$ for $\sigma>\frac{1}{2}$. Hence, the above semidiscrete DG formulation (2.16) is consistent with the original continuous problem (2.6).

In (2.19) the function a penalizes the jumps of $u$ and $v$ over the faces of $\mathcal{T}_{h}$. It is referred to as the IP stabilization function and is defined as follows. We first introduce the function $\mathrm{h}$ by

$$
\left.\mathrm{h}\right|_{F}= \begin{cases}\min \left\{h_{K}, h_{K^{\prime}}\right\}, & F \in \mathcal{F}_{h}^{\mathcal{I}}, F=\partial K \cap \partial K^{\prime}, \\ h_{K}, & F \in \mathcal{F}_{h}^{\mathcal{B}}, F=\partial K \cap \partial \Omega .\end{cases}
$$

For $x \in F$, we further define $\mathrm{c}$ by

$$
\left.\mathrm{c}\right|_{F}(x)= \begin{cases}\max \left\{\left.c\right|_{K}(x),\left.c\right|_{K^{\prime}}(x)\right\}, & F \in \mathcal{F}_{h}^{\mathcal{I}}, F=\partial K \cap \partial K^{\prime}, \\ \left.c\right|_{K}(x), & F \in \mathcal{F}_{h}^{\mathcal{B}}, F=\partial K \cap \partial \Omega .\end{cases}
$$

Then, on each $F \in \mathcal{F}_{h}$, we set

$$
\left.\mathrm{a}\right|_{F}:=\alpha \mathrm{c}^{2} \mathrm{~h}^{-1},
$$

where $\alpha$ is a positive parameter independent of the local mesh sizes and the coefficient $c$. There exists a threshold value $\alpha_{\min }>0$ which depends only on the shape regularity of the mesh and the approximation order $\ell$ such that for $\alpha \geq \alpha_{\min }$ the DG bilinear form $a_{h}$ is coercive and, hence, the discretization stable [3]. Throughout the rest of the paper we shall assume that $\alpha \geq \alpha_{\min }$ so that the semidiscrete problem (2.16)-(2.18) has a unique solution which converges with optimal order [23].

The semidiscrete IP-DG formulation (2.16)-(2.18) is equivalent to the secondorder system of ordinary differential equations

$$
\begin{aligned}
M \frac{d^{2} y}{d t^{2}}+K y & =F, \\
y(0)=y_{0}, \quad \frac{d y}{d t}(0) & =\dot{y}_{0} .
\end{aligned}
$$

The $N \times N$ mass matrix $M$, with entries $M_{i j}=\left(\phi_{i}, \phi_{j}\right)$, again is symmetric positive definite. Yet because individual elements decouple, $M$ is also block-diagonal, with block size equal to the number of degrees of freedom per element. Thus, it can be inverted at very low computational cost. In fact, for a judicious choice of (locally orthogonal) shape functions, the mass matrix is diagonal and, therefore, the resulting time-marching scheme truly explicit.

Remark 2.1. Because the matrices $M$ and $K$ are symmetic, either for the symmetric IP-DG or for the standard continuous Galerkin discretization, the two semidiscrete formulations (2.13)-(2.14) and (2.21)-(2.22) conserve the (discrete) energy

$$
E_{h}(t):=\frac{1}{2}\left\{\left\langle M y^{\prime}(t) y^{\prime}(t)\right\rangle+\langle K y(t), y(t)\rangle\right\}
$$

when $F=0$. Here, the angular brackets denote the standard Euclidean inner product on $\mathbb{R}^{N}$. If the underlying bilinear form $a$ is strictly coercive, so are the two discrete bilinear forms $a_{h}$, either for the DG discretization with $\alpha \geq \alpha_{\min }$ or for the continuous Galerkin discretization with (or without) mass lumping. For $f=0$, conservation of 
energy then implies that the solution remains bounded for all time. However, when the underlying bilinear form $a$ is not (strictly) coercive, as in the presence of Neumann or periodic boundary conditions, for instance, the elliptic partial differential operator will have a (simple) zero eigenvalue (with constant eigenfunction). Then, the wave equation admits linear growth in time, although the (continuous or discrete) energy remains constant. Nonetheless, if the numerical method is consistent, stability (with respect to the energy) will imply convergence on any finite time interval. Moreover, since all time integration schemes considered here are at least second-order accurate, the numerical time integration of the linearly growing zeroth eigenmode will actually be exact.

3. Local time stepping. We consider the semidiscrete wave equation

$$
M \frac{d^{2} y}{d t^{2}}+K y=0
$$

where $M$ is an $N \times N$ symmetric positive definite (sparse) matrix and $K$ is an $N \times N$ symmetric positive semidefinite (sparse) matrix. Moreover, we assume that $M^{\frac{1}{2}}$ can be explicitly computed and inverted at low cost, as in the case when $M$ is (block-) diagonal, for instance. Next, we multiply (3.1) by $M^{-\frac{1}{2}}$ to obtain

$$
\frac{d^{2} z}{d t^{2}}+M^{-\frac{1}{2}} K M^{-\frac{1}{2}} z=0
$$

Let $A$ denote the matrix $M^{-\frac{1}{2}} K M^{-\frac{1}{2}}$, which is also sparse and symmetric positive semidefinite. Then, we rewrite (3.2) as

$$
\frac{d^{2} z}{d t^{2}}+A z=0
$$

For any $f \in \mathcal{C}^{1}$ we have

$$
f(t+\Delta t)-2 f(t)+f(t-\Delta t)=-\Delta t^{2} \int_{-1}^{1}(1-|\theta|) f^{\prime \prime}(t+\theta \Delta t) d \theta .
$$

Hence, the (exact) solution $z(t)$ of (3.3) satisfies

$$
z(t+\Delta t)-2 z(t)+z(t-\Delta t)=-\Delta t^{2} \int_{-1}^{1}(1-|\theta|) A z(t+\theta \Delta t) d \theta .
$$

3.1. Second-order local time-stepping method. The integral on the right side of (3.5) represents a weighted average of $A z(s)$ over the interval $[t-\Delta t, t+\Delta t]$, which needs to be approximated in any numerical algorithm. For instance, if we simply replace $A z(t+\theta \Delta t)$ by $A z(t)$ in (3.5) and evaluate the remaining $\theta$-dependent integral, we obtain the well-known second-order leap-frog scheme with time step $\Delta t$,

$$
z_{n+1}-2 z_{n}+z_{n-1}=-\Delta t^{2} A z_{n}, \quad z_{n} \simeq z\left(t_{n}\right),
$$

which, however, would require $\Delta t$ to be comparable in size to the smallest elements in the mesh. Instead, we now split the vector $z(t)$ in two parts:

$$
z(t)=(I-P) z(t)+P z(t)=z^{[\text {coarse }]}(t)+z^{[\text {fine }]}(t) .
$$


The projection matrix $P$ is diagonal: its diagonal entries, equal to zero or one, identify the unknowns associated with the locally refined region, that is where smaller time steps are needed. To circumvent the severe CFL restriction on $\Delta t$ in (3.6), we shall treat $z^{[\text {fine] }}(t)$ differently from $z^{[\text {coarse }]}(t)$ in

$$
\begin{aligned}
z(t+ & \Delta t)-2 z(t)+z(t-\Delta t) \\
& =-\Delta t^{2} \int_{-1}^{1}(1-|\theta|) A\left[z^{[\text {coarse }]}(t+\theta \Delta t)+z^{[\text {fine }]}(t+\theta \Delta t)\right] d \theta .
\end{aligned}
$$

Following $[29,35]$, we approximate the integrand in (3.7) as

$$
A\left[z^{[\text {coarse }]}(t+\theta \Delta t)+z^{[\mathrm{fine}]}(t+\theta \Delta t)\right] \simeq A z^{[\text {coarse }]}(t)+A P \tilde{z}(\theta \Delta t)
$$

where $\tilde{z}(\tau)$ solves the differential equation

$$
\left\{\begin{array}{l}
\frac{d^{2} \tilde{z}}{d \tau^{2}}(\tau)=-A(I-P) z(t)-A P \tilde{z}(\tau), \\
\tilde{z}(0)=z(t), \quad \frac{d \tilde{z}}{d \tau}(0)=\nu
\end{array}\right.
$$

and $\nu$ will be specified below. Thus,

$$
z(t+\Delta t)-2 z(t)+z(t-\Delta t) \simeq-\Delta t^{2} \int_{-1}^{1}(1-|\theta|)[A(I-P) z(t)+A P \tilde{z}(\theta \Delta t)] d \theta
$$

Note that $A$ and $P$ do not commute. Since $\tilde{z}$ solves (3.8), we deduce again from (3.4) that

$$
\tilde{z}(\Delta t)-2 \tilde{z}(0)+\tilde{z}(-\Delta t)=-\Delta t^{2} \int_{-1}^{1}(1-|\theta|)[A(I-P) z(t)+A P \tilde{z}(\theta \Delta t)] d \theta
$$

From the comparison of (3.9) and (3.10) we infer that

$$
z(t+\Delta t)-2 z(t)+z(t-\Delta t) \simeq \tilde{z}(\Delta t)-2 \tilde{z}(0)+\tilde{z}(-\Delta t) .
$$

The quantity $\tilde{z}(\Delta t)-2 \tilde{z}(0)+\tilde{z}(-\Delta t)$ does not depend on the value of $\nu$. Indeed, let $g(t)=A(I-P) z(t)$ and $C=(A P)^{1 / 2}$. Then, the solution of (3.8) is given by

$$
\tilde{z}(\tau)=C^{-1} \sin (C \tau) \nu+\cos (C \tau) z(t)-C^{-2}[I-\cos (C \tau)] g(t),
$$

from which we immediately conclude that

$$
\tilde{z}(\tau)-2 \tilde{z}(0)+\tilde{z}(-\tau)=2[\cos (C \tau)-I] z(t)-2 C^{-2}[I-\cos (C \tau)] g(t),
$$


which is independent of $\nu$. Thus, we may choose $\nu=0$, which further implies that $\tilde{z}(\tau)=\tilde{z}(-\tau)$ so that (3.11) becomes

$$
z(t+\Delta t)-2 z(t)+z(t-\Delta t) \simeq 2(\tilde{z}(\Delta t)-\tilde{z}(0))
$$

or, equivalently,

$$
z(t+\Delta t)+z(t-\Delta t) \simeq 2 \tilde{z}(\Delta t) .
$$

Hence, we shall approximate the right side of (3.13) by solving (3.8) on $[0, \Delta t]$ and then use (3.13) to compute $z(t+\Delta t)$. Since the first term on the right side of (3.8) does not depend on $\tau$, the (high-frequency, oscillatory) evolution of $\tilde{z}(\tau)$ is solely determined by the second term $A P \tilde{z}(\tau)$, which involves only the unknowns associated with the nonzero entries in $P$. If those nonzero entries occupy only a small fraction of all unknowns, the additional effort from solving (3.8) will be small since $A$ is sparse. Clearly, in doing so we must also ensure that the overall numerical scheme remains second-order accurate in time, as we shall show below.

In summary, the local time-stepping algorithm for the solution of (3.1) computes $z_{n+1} \simeq z(t+\Delta t)$, given $z_{n}$ and $z_{n-1}$, as follows.

Algorithm 3.1.

1. Set $w=A(I-P) z_{n}$ and $\tilde{z}_{0}=z_{n}$.

2. Compute $\tilde{z}_{1 / p}=\tilde{z}_{0}-\frac{1}{2}\left(\frac{\Delta t}{p}\right)^{2}\left(w+A P \tilde{z}_{0}\right)$.

3. For $m=1, \ldots, p-1$, compute

$$
\tilde{z}_{(m+1) / p}=2 \tilde{z}_{m / p}-\tilde{z}_{(m-1) / p}-\left(\frac{\Delta t}{p}\right)^{2}\left(w+A P \tilde{z}_{m / p}\right) .
$$

4. Compute $z_{n+1}=-z_{n-1}+2 \tilde{z}_{1}$.

Here, steps 1-3 correspond to the numerical solution of (3.8) with $\nu=0$ until $\tau=\Delta t$ using the leap-frog scheme with the local time step $\Delta \tau=\Delta t / p$. In fact, any other second-order method, either explicit or implicit, could be used there instead. For $P=0$, that is without any local time stepping, we have

$$
\tilde{z}_{1}=\tilde{z}(\Delta t)=\left(I-\frac{\Delta t^{2}}{2} A\right) z(t),
$$

and, hence, we recover the standard leap-frog scheme (3.6). If the fraction of nonzero entries in $P$ is small, the overall cost will be dominated by the computation of $w$, which requires a single multiplication by $A(I-P)$ per time step $\Delta t$. All further matrix-vector multiplications by $A P$ involve only those unknowns that are associated with the smaller, locally refined region. In addition, since $A$ is sparse, every update in step 3 affects only those unknowns that lie inside the refined region or immediately next to it.

To establish the accuracy and stability of the above local time-stepping scheme, we shall now show how to rewrite it in "leap-frog manner." To do so, we first need the following technical result.

Lemma 3.2. For $m \geq 2, \tilde{z}_{m / p}$ defined by Algorithm 3.1 satisfies

$$
\tilde{z}_{m / p}=z_{n}-\frac{m^{2}}{2}\left(\frac{\Delta t}{p}\right)^{2} A z_{n}+\sum_{j=1}^{m-1}\left(\frac{\Delta t}{p}\right)^{2(j+1)} \alpha_{j}^{m}(A P)^{j} A z_{n},
$$


where the constants $\alpha_{j}^{m}$ are given by

$$
\left\{\begin{array}{l}
\alpha_{1}^{2}=\frac{1}{2}, \quad \alpha_{1}^{3}=3, \quad \alpha_{2}^{3}=-\frac{1}{2} \\
\alpha_{1}^{m+1}=\frac{m^{2}}{2}+2 \alpha_{1}^{m}-\alpha_{1}^{m-1}, \\
\alpha_{j}^{m+1}=2 \alpha_{j}^{m}-\alpha_{j}^{m-1}-\alpha_{j-1}^{m}, \quad j=2 \ldots m-2, \\
\alpha_{m-1}^{m+1}=2 \alpha_{m-1}^{m}-\alpha_{m-2}^{m}, \\
\alpha_{m}^{m+1}=-\alpha_{m-1}^{m} .
\end{array}\right.
$$

Proof. The proof is by induction on $m$.

We first show that (3.15) holds for $m=2$. Since

$$
\tilde{z}_{1 / p}=z_{n}-\frac{1}{2}\left(\frac{\Delta t}{p}\right)^{2} A z_{n}
$$

we immediately find that

$$
\tilde{z}_{2 / p}=z_{n}-2\left(\frac{\Delta t}{p}\right)^{2} A z_{n}+\frac{1}{2}\left(\frac{\Delta t}{p}\right)^{4} A P A z_{n} .
$$

Hence, (3.15) holds with $\alpha_{1}^{2}=1 / 2$.

Next, let (3.15) hold for $m$. Then,

$$
\begin{aligned}
\tilde{z}_{(m+1) / p}= & 2\left(z_{n}-\frac{m^{2}}{2}\left(\frac{\Delta t}{p}\right)^{2} A z_{n}+\sum_{j=1}^{m-1}\left(\frac{\Delta t}{p}\right)^{2(j+1)} \alpha_{j}^{m}(A P)^{j} A z_{n}\right) \\
& -\left(z_{n}-\frac{(m-1)^{2}}{2}\left(\frac{\Delta t}{p}\right)^{2} A z_{n}\right. \\
& \left.+\sum_{j=1}^{m-2}\left(\frac{\Delta t}{p}\right)^{2(j+1)} \alpha_{j}^{m-1}(A P)^{j} A z_{n}\right) \\
& -\left(\frac{\Delta t}{p}\right)^{2}\left(A(I-P) z_{n}+A P\left[z_{n}-\frac{m^{2}}{2}\left(\frac{\Delta t}{p}\right)^{2} A z_{n}\right.\right. \\
& \left.\left.+\sum_{j=1}^{m-1}\left(\frac{\Delta t}{p}\right)^{2(j+1)} \alpha_{j}^{m}(A P)^{j} A z_{n}\right]\right)^{2},
\end{aligned}
$$

which after some algebra simplifies to

$$
\begin{aligned}
\tilde{z}_{(m+1) / p}= & z_{n}-\left(m^{2}-\frac{(m-1)^{2}}{2}+1\right)\left(\frac{\Delta t}{p}\right)^{2} A z_{n}+\frac{m^{2}}{2}\left(\frac{\Delta t}{p}\right)^{4} A P A z_{n} \\
& +2 \sum_{j=1}^{m-1}\left(\frac{\Delta t}{p}\right)^{2(j+1)} \alpha_{j}^{m}(A P)^{j} A z_{n} \\
& -\sum_{j=1}^{m-2}\left(\frac{\Delta t}{p}\right)^{2(j+1)} \alpha_{j}^{m-1}(A P)^{j} A z_{n} \\
& -\sum_{j=1}^{m-1}\left(\frac{\Delta t}{p}\right)^{2(j+2)} \alpha_{j}^{m}(A P)^{j+1} A z_{n} .
\end{aligned}
$$


Rearranging terms we then find

$$
\begin{aligned}
\tilde{z}_{(m+1) / p}= & z_{n}-\frac{(m+1)^{2}}{2}\left(\frac{\Delta t}{p}\right)^{2} A z_{n}+\left(\frac{\Delta t}{p}\right)^{4}\left(\frac{m^{2}}{2}+2 \alpha_{1}^{m}-\alpha_{1}^{m-1}\right) A P A z_{n} \\
& +\sum_{j=2}^{m-2}\left(\frac{\Delta t}{p}\right)^{2(j+1)}\left(2 \alpha_{j}^{m}-\alpha_{j}^{m-1}-\alpha_{j-1}^{m}\right)(A P)^{j} A z_{n} \\
& +\left(\frac{\Delta t}{p}\right)^{2 m}\left(2 \alpha_{m-1}^{m}-\alpha_{m-2}^{m}\right)(A P)^{m-1} A z_{n} \\
& -\left(\frac{\Delta t}{p}\right)^{2(m+1)} \alpha_{m-1}^{m}(A P)^{m} A z_{n},
\end{aligned}
$$

which yields (3.15) with $\alpha_{j}^{m}$ as in (3.16).

As a consequence, we can rewrite the above local time-stepping algorithm in "leap-frog manner."

Proposition 3.3. The local time-stepping Algorithm 3.1 is equivalent to

$$
z_{n+1}=2 z_{n}-z_{n-1}-\Delta t^{2} A_{p} z_{n},
$$

where $A_{p}$ is defined by

$$
A_{p}=A-\frac{2}{p^{2}} \sum_{j=1}^{p-1}\left(\frac{\Delta t}{p}\right)^{2 j} \alpha_{j}^{p}(A P)^{j} A
$$

and the constants $\alpha_{j}^{p}$ are given by (3.16). This scheme is second-order accurate. Furthermore, the matrix $A_{p}$ is symmetric.

Proposition 3.3 is crucial for the accuracy and stability analysis below. However, the actual implementation of the local time-stepping scheme follows Algorithm 3.1; in particular, neither $A_{p}$ nor the constants $\alpha_{j}^{p}$ are ever used in practice.

Proof. Recall that $z_{n+1}=-z_{n-1}+2 \tilde{z}_{1}$. We now use (3.15) with $m=p$ to replace $\tilde{z}_{1}$. This yields

$$
z_{n+1}=-z_{n-1}+2 z_{n}-\Delta t^{2} A z_{n}+2\left(\frac{\Delta t}{p}\right)^{2} \sum_{j=1}^{p-1}\left(\frac{\Delta t}{p}\right)^{2 j} \alpha_{j}^{p}(A P)^{j} A z_{n}
$$

which corresponds to (3.17) with $A_{p}$ as in (3.18).

To prove consistency we now rewrite (3.19) as

$$
\frac{z_{n+1}-2 z_{n}+z_{n-1}}{\Delta t^{2}}-\frac{2}{p^{2}} \sum_{j=1}^{p-1}\left(\frac{\Delta t}{p}\right)^{2 j} \alpha_{j}^{p}(A P)^{j} A z_{n}=-A z_{n} .
$$

Since

$$
\frac{d^{2} z}{d t^{2}}(t)=\frac{z(t+\Delta t)-2 z(t)+z(t-\Delta t)}{\Delta t^{2}}+O\left(\Delta t^{2}\right)
$$

and

$$
\sum_{j=1}^{p-1}\left(\frac{\Delta t}{p}\right)^{2 j} \alpha_{j}^{p}(A P)^{j} A z(t)=O\left(\Delta t^{2}\right)
$$

we conclude that the local time-stepping scheme is second-order accurate in time. 
Finally, as the matrices $A$ and $P$ are symmetric, we have

$$
\left((A P)^{j} A\right)^{\top}=A(P A)^{j}=(A P)^{j} A, \quad j \geq 1 .
$$

Therefore, the matrix $A_{p}$ is symmetric, too.

3.2. Energy conservation. For symmetric $A$ it is well-known that the standard leap-frog scheme (3.6) conserves the discrete energy

$$
E^{n+\frac{1}{2}}=\frac{1}{2}\left[\left\langle\left(I-\frac{\Delta t^{2}}{4} A\right) \frac{z_{n+1}-z_{n}}{\Delta t}, \frac{z_{n+1}-z_{n}}{\Delta t}\right\rangle+\left\langle A \frac{z_{n+1}+z_{n}}{2}, \frac{z_{n+1}+z_{n}}{2}\right\rangle\right] .
$$

Here, $E^{n+\frac{1}{2}} \simeq E_{h}\left(t_{n+\frac{1}{2}}\right)$ (see Remark 2.1) and the angular brackets denote the standard Euclidean inner product on $\mathbb{R}^{N}$. Since $A$ is symmetric, so is the quadratic form in (3.20). Moreover, for sufficiently small $\Delta t$ it is also positive semidefinite and, hence, yields a true energy. Precisely this restriction on $\Delta t$ corresponds to the CFL stability condition of the leap-frog method and guarantees its numerical stability.

From Proposition 3.3 we know that the local time-stepping algorithm is equivalent to (3.6) with $A_{p}$ replaced by $A$. Since $A_{p}$ is also symmetric, we can inmmediately exhibit the following conserved quantity.

Proposition 3.4. The second-order local time-stepping scheme (Algorithm (3.1)) conserves the discrete energy

$$
E^{n+\frac{1}{2}}=\frac{1}{2}\left[\left\langle\left(I-\frac{\Delta t^{2}}{4} A_{p}\right) \frac{z_{n+1}-z_{n}}{\Delta t}, \frac{z_{n+1}-z_{n}}{\Delta t}\right\rangle+\left\langle A_{p} \frac{z_{n+1}+z_{n}}{2}, \frac{z_{n+1}+z_{n}}{2}\right\rangle\right] .
$$

The energy in (3.21) corresponds to the energy conserved by the leap-frog scheme with $A$ replaced by $A_{p}$.

The local time-stepping Algorithm 3.1 conserves the energy $E^{n+\frac{1}{2}}$ in (3.21), which guarantees stability if and only if both terms of $E^{n+\frac{1}{2}}$ are positive semidefinite or, equivalently, if and only if the matrices $\left(I-\frac{\Delta t^{2}}{4} A_{p}\right)$ and $A_{p}$ are both positive semidefinite. Hence, if $\lambda_{\min }$ and $\lambda_{\max }$ denote the smallest and largest eigenvalues of $A_{p}$, respectively, the numerical scheme will be stable if and only if

$$
0 \leq \frac{\Delta t^{2}}{4} \lambda_{\min } \leq \frac{\Delta t^{2}}{4} \lambda_{\max } \leq 1
$$

For $p=1$, we have $A_{p}=A$, and, thus, we recover the well-known CFL condition of the standard leap-frog (LF) scheme:

$$
\Delta t \leq \frac{2}{\sqrt{\lambda_{\max }}}=\Delta t_{L F}
$$

For $p>1$, the matrix $A_{p}$ explicitly depends on $\Delta t$, and so do its eigenvalues. Moreover, as the eigenvectors of $A$ and $A_{p}$ generally do not coincide, the analytic derivation of a CFL condition is not obvious. Instead, we shall perform a systematic numerical study of the eigenvalues of $\frac{\Delta t^{2}}{4} A_{p}$ in the following typical situation.

3.3. Stability and CFL condition: Numerical study. We consider the onedimensional wave equation with constant wave speed $c=1$ on the interval $\Omega=[0 ; 6]$ with periodic boundary conditions. Next, we divide $\Omega$ into three equal parts. The left and right intervals, $[0 ; 2]$ and $[4 ; 6]$, respectively, are discretized with an equidistant 


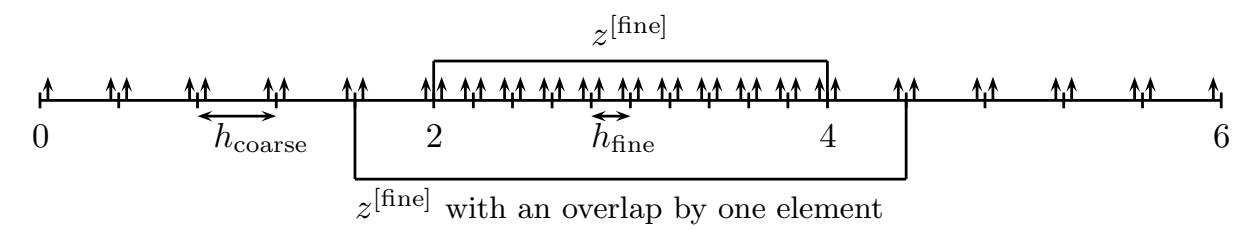

FIG. 3.1. IP-DG $\mathcal{P}^{1}$-elements. The computational mesh and the associated degrees of freedom, indicated by small arrows.
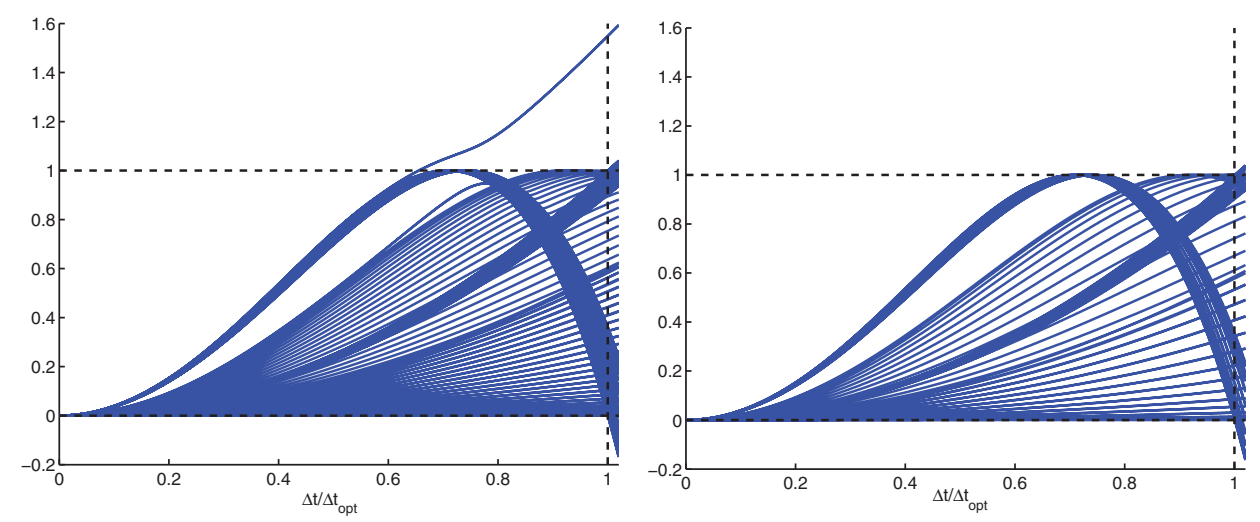

FIG. 3.2. IP-DG with $\mathcal{P}^{1}$-elements. The eigenvalues of $\left(\Delta t^{2} / 4\right) A_{p}$ : without overlap (left); with an overlap by one element (right).

mesh of size $h_{\text {coarse }}$, whereas the interval $[2 ; 4]$ is discretized with an equidistant mesh of size $h_{\text {fine }}=h_{\text {coarse }} / p$. Hence, the two outer intervals correspond to the coarse region and the inner interval $[2 ; 4]$ to the refined region-see Figure 3.1.

For every time step $\Delta t$, we shall take $p$ steps of size $\Delta \tau=\Delta t / p$ in the refined region. In the absence of local refinement, i.e., $p=1$, the mesh is equidistant throughout $\Omega$. Then, the (local) time-stepping algorithm corresponds to the standard leap-frog method and we denote by $\Delta t_{L F}$ the largest time step allowed. For $p \geq 2$, we let $\Delta t_{p}$ denote the maximal time step of Algorithm 3.1. If $\Delta t_{p}=\Delta t_{L F}$, the local timestepping algorithm imposes no further restriction on $\Delta t$ and we then shall call the CFL condition of the new scheme optimal.

First, we consider the IP-DG discretization with $\mathcal{P}^{1}$-elements and (small) penalization $\alpha=2$. We choose $h_{\text {coarse }}=0.2$, which yields the maximal time step $\Delta t_{L F}=0.55 h_{\text {coarse }}=0.11$ for $p=1$. Now, we refine by a factor $p=2$ those elements that lie inside the interval $[2,4]$, that is $h_{\text {fine }}=0.1$, and set to one all corresponding entries in $P$. Hence, for every time step $\Delta t$, we shall take two steps of size $\Delta \tau=\Delta t / 2$ in the refined region.

To determine the range of values $\Delta t$ for which the local time-stepping scheme is stable, we display the eigenvalues of $\left(\Delta t^{2} / 4\right) A_{p}$ for varying $\Delta t / \Delta t_{L F}$-recall that $A_{p}$ also depends on $\Delta t$. The numerical scheme is stable for any particular $\Delta t$ if all corresponding eigenvalues lie between zero and one; otherwise, it is unstable. Since the smallest eigenvalue actually never dips below zero, it does not affect the stability here. As shown in the left frame of Figure 3.2, the largest time step allowed is only about $60 \%$ of $\Delta t_{L F}$; hence, the gain over a straightforward reduction of the (global) time step by a factor of two is rather modest. 

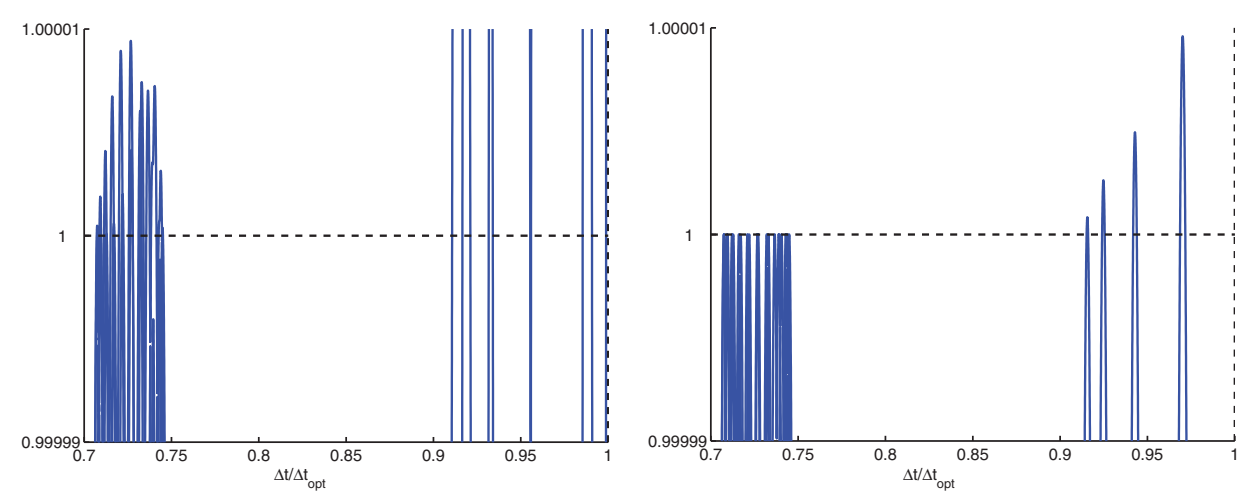

Fig. 3.3. IP-DG $\mathcal{P}^{1}$-elements. The eigenvalues of $\left(\Delta t^{2} / 4\right) A_{p}$ : overlap by one element (left); overlap by two elements (right). The vertical scale is strongly magnified: $0.99999<\lambda_{\max }<1.00001$.

TABLE 3.1

IP-DG $\mathcal{P}^{1}$-elements. The largest eigenvalue of $\left(\Delta t^{2} / 4\right) A_{p}$ with $\Delta t=\Delta t_{\text {opt }}$ and $h_{\text {fine }}=$ $h_{\text {coarse }} / p$ for an overlap by one element.

\begin{tabular}{|c|c|c|c|c|c|}
\hline & \multicolumn{3}{|c|}{$\mathrm{p}$} \\
\hline$h_{\text {coarse }}$ & 2 & 3 & 4 & 10 & 13 \\
\hline 0.5 & 1.0002 & 0.9912 & 0.9983 & 1.0003 & 1.0005 \\
\hline 0.2 & 1.0009 & 0.9999 & 1.0003 & 1.0002 & 1.0002 \\
\hline 0.1 & 1.0006 & 1.00001 & 0.9997 & 0.9999 & 0.9999 \\
\hline 0.05 & 1.0005 & 1.0001 & 0.9999 & 1.00006 & 0.9999 \\
\hline 0.025 & 1.0005 & 1.00009 & 1.00005 & 1.00002 & 1.00002 \\
\hline
\end{tabular}

To allow for larger time steps, we now slightly enlarge the set of unknowns where a local time step is used by also including those degrees of freedom that are associated with elements directly adjacent to the refined region. By setting the corresponding entries in $P$ to one, we easily realize this overlap by one element in $z^{\text {[fine] }}$. In the right frame of Figure 3.2 we observe that all eigenvalues now lie essentially between zero and one. However, a hundred thousandfold magnification of that same figure, shown in the left frame of Figure 3.3, reveals that some eigenvalues still barely transgress the strict stability limit at one. Further extension of the overlap by one additional element removes all unstable values below $0.9 \Delta t_{L F}$, as shown in the right frame of Figure 3.3, while four narrow bands of (barely) unstable values between $0.91 \leq \Delta t / \Delta t_{L F} \leq 0.98$ remain. Here, we shall not attempt to elucidate that peculiar and somewhat sensitive behavior, due to weak resonances caused by the underlying regular, one-dimensional grid.

Instead, we now address the question of whether the local time-stepping scheme is stable for the maximal time step $\Delta t=\Delta t_{L F}$. In Table 3.1 we list the corresponding maximal eigenvalue of $\left(\Delta t^{2} / 4\right) A_{p}$ for an overlap by one element with different mesh sizes $h_{\text {coarse }}=0.5,0.2,0.1,0.05,0.025$ and different $p=2,3,4,10,13$; here, the maximal eigenvalue typically is greater than one and the local time-stepping scheme, therefore, unstable for the optimal time step. Yet with an overlap by two elements, the maximal eigenvalues listed in Table 3.2 now always lie below one, independently of $h_{\text {coarse }}$ and $p$; hence, for an overlap by two elements, Algorithm 3.1 is always stable for the optimal time step.

Next, we repeat the above experiment with standard $\mathcal{P}^{1}$ continuous, piecewise linear finite elements with mass lumping. Then, the CFL condition for the classical leap-frog scheme is $\Delta t_{L F}=h_{\text {coarse }}=0.2$. In contrast to the IP-DG FEM, a degree 
TABLE 3.2

IP-DG $\mathcal{P}^{1}$-elements. The largest eigenvalue of $\left(\Delta t^{2} / 4\right) A_{p}$ with $\Delta t=\Delta t_{L F}$ and $h_{\text {fine }}=$

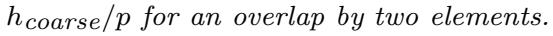

\begin{tabular}{|c|c|c|c|c|c|}
\hline & \multicolumn{5}{|c|}{$\mathrm{p}$} \\
\hline$h_{\text {coarse }}$ & 2 & 3 & 4 & 10 & 13 \\
\hline 0.5 & 0.9981 & 0.9902 & 0.9983 & 0.9997 & 0.9999 \\
\hline 0.2 & 0.9998 & 0.9994 & 0.9999 & 0.9999 & 0.9999 \\
\hline 0.1 & 0.9998 & 0.9999 & 0.9996 & 0.9999 & 0.9999 \\
\hline 0.05 & 0.9999 & 0.9999 & 0.9999 & 0.9999 & 0.9999 \\
\hline 0.025 & 0.9999 & 0.9999 & 0.9999 & 0.9999 & 0.9999 \\
\hline
\end{tabular}

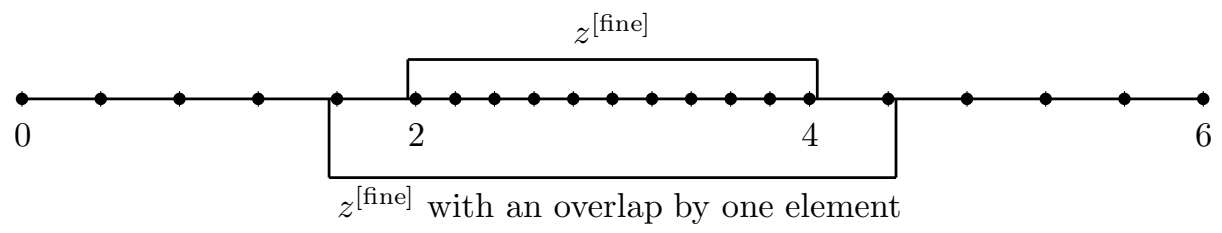

FIG. 3.4. The nodes in $z^{[\text {fine }]}$ for continuous $\mathcal{P}^{1}$-elements.

TABLE 3.3
Continuous $\mathcal{P}^{1}-F E$. The largest eigenvalue of $\frac{\Delta t^{2}}{4} A_{p}$ at $\Delta t=\Delta t_{L F}$ for an overlap by one element.

\begin{tabular}{|c|c|c|c|c|c|}
\hline & \multicolumn{5}{|c|}{$\mathrm{p}$} \\
\hline$h_{\text {coarse }}$ & 2 & 3 & 4 & 10 & 13 \\
\hline 0.5 & 0.9828 & 0.9792 & 0.9993 & 0.9999 & 0.9999 \\
\hline 0.2 & 0.9969 & 0.9962 & 0.9999 & 0.9999 & 0.9999 \\
\hline 0.1 & 0.9992 & 0.9991 & 0.9999 & 0.9999 & 0.9999 \\
\hline 0.05 & 0.9998 & 0.9998 & 0.9999 & 0.9999 & 0.9999 \\
\hline 0.025 & 0.9999 & 0.9999 & 0.9999 & 0.9999 & 0.9999 \\
\hline
\end{tabular}

of freedom can now belong to both a fine and a coarse element; hence, any degree of freedom at the interface between the two subregions is automatically included in $z^{[\text {fine] }}$, as shown in Figure 3.4.

In Table 3.3 we list the maximal eigenvalue of $\frac{\Delta t^{2}}{4} A_{p}$ at $\Delta t=\Delta t_{L F}$ for an overlap by a single element for different mesh sizes $h_{\text {coarse }}=0.5,0.2,0.1,0.05,0.025$ and different $p=2,3,4,10,13$. Here, we find that an overlap by only one element already leads to a stable scheme for the optimal time step.

In summary, a slight extension (overlap) of the region where local time steps are used into that part of the mesh immediately adjacent to the refined region typically improves the stability of the time-stepping scheme. Moreover, our numerical results suggest that an overlap by one element for the $\mathcal{P}^{1}$ continuous $\mathrm{FE}$ discretization (with mass lumping), or by two elements for the IP-DG discretization, permits the use of the maximal (optimal) time step $\Delta t_{L F}$, dictated by the leap-frog method in the coarse region.

4. High order local time stepping. We shall now show how to extend the second-order local time-stepping method from section 3 to arbitrarily high accuracy. First, we develop in detail a fourth-order local time-stepping scheme and again exhibit a conserved discrete energy. Then, we consider the general case of arbitrary (even) order. By including a small overlap into the surrounding coarser region we show via numerical experiments that both the fourth- and sixth-order local time-stepping schemes are stable for the optimal time step dictated by the coarse mesh size. 
4.1. Fourth-order local time stepping. A fourth-order extension of the leapfrog scheme for (3.3) is provided by the modified equation (ME) approach $[7,18,2,41]$. Thus, we replace $A z(t+\theta \Delta t)$ in (3.5) by the leading terms in its Taylor expansion

$$
A z(t+\theta \Delta t) \simeq A\left(z(t)+\theta \Delta t z^{\prime}(t)+\frac{\theta^{2} \Delta t^{2}}{2} z^{\prime \prime}(t)\right) .
$$

By integrating in (3.5) the resulting polynomial in $\theta$, the first derivative of $z$ vanishes; since $z^{\prime \prime}(t)=-A z(t)$, we, thus, obtain the ME scheme:

$$
\frac{z_{m+1}-2 z_{m}+z_{m-1}}{\Delta t^{2}}=-A z_{m}+\frac{\Delta t^{2}}{12} A^{2} z_{m}, \quad z_{m} \simeq z\left(t_{m}\right) .
$$

In contrast to standard fourth-order Runge-Kutta schemes, the ME approach requires only two multiplications with $A$ per time step. Its CFL condition follows from the CFL condition of the leap-frog scheme [14],

$$
\Delta t_{M E} \leq \frac{2 \sqrt{3}}{\sqrt{\lambda_{\max }}}=\sqrt{3} \Delta t_{L F}
$$

As the maximal time step allowed by the ME approach is about $70 \%$ larger than that of the leap-frog scheme, the additional work needed for the improved accuracy is quite small. Clearly, high-order schemes of arbitrary (even) order can be obtained by using additional terms in the Taylor expansion of $A z(t+\theta \Delta t)$. Recently, Gilbert and Joly derived new time-stepping methods that maximize the CFL condition for a given order of accuracy [21].

Following [37], we expand in (3.7) $z^{\text {[coarse] }}$ in the Taylor series as

$$
z^{[\text {coarse }]}(t+\theta \Delta t)=z^{[\text {coarse }]}(t)+\theta \Delta t \frac{d z^{[\text {coarse }]}}{d t}(t)+\frac{\theta^{2} \Delta t^{2}}{2} \frac{d^{2} z^{[\text {coarse }]}}{d t^{2}}(t)+O\left(\Delta t^{3}\right) .
$$

Since

$$
\frac{d^{2} z^{[\text {coarse }]}}{d t^{2}}(t)=(I-P) \frac{d^{2} z}{d t^{2}}(t)=-(I-P) A z(t)
$$

we find that

$$
z^{[\text {coarse] }}(t+\theta \Delta t) \simeq(I-P) z(t)+\theta \Delta t(I-P) \frac{d z}{d t}(t)-\frac{\theta^{2} \Delta t^{2}}{2}(I-P) A z(t) .
$$

Next, we use (4.2) in (3.7) to replace $z^{\text {[coarse] }}$, which yields

$$
\begin{aligned}
z(t+\Delta t)-2 z(t)+z(t-\Delta t) \simeq & -\Delta t^{2} A(I-P) z(t)+\frac{\Delta t^{4}}{12} A(I-P) A z(t) \\
& -\Delta t^{2} \int_{-1}^{1}(1-|\theta|) A z^{[\mathrm{fine}]}(t+\theta \Delta t) d \theta
\end{aligned}
$$

Hence, if $P=0$, we recover the standard ME scheme (4.1). 
Similar to section 3.1, we shall now approximate the right-hand side of (4.3) by solving the following differential equation for $\tilde{z}(\tau)$ :

$$
\left\{\begin{array}{l}
\frac{d^{2} \tilde{z}}{d \tau^{2}}=-A(I-P) z(t)+\frac{\tau^{2}}{2} A(I-P) A z(t)-A P \tilde{z}(\tau), \\
\tilde{z}(0)=z(t), \quad \frac{d \tilde{z}}{d \tau}(0)=0 .
\end{array}\right.
$$

Again, by symmetry, we have

$$
z(t+\Delta t)+z(t-\Delta t) \simeq 2 \tilde{z}(\Delta t) .
$$

Thus, we approximate the right side of (4.3) by solving (4.4) on $[0, \Delta t]$ and then use (4.5) to compute $z(t+\Delta t)$. The last term on the right side of (4.3) explicitly depends on $\tilde{z}(\tau)$, but it involves only the unknowns associated with the nonzero entries in $P$. If those nonzero entries occupy only a small fraction of all unknowns, the additional effort from solving (4.4) will be small. Clearly, in doing so we must ensure that the overall numerical scheme remains fourth-order accurate in time.

In summary, the fourth-order local time-stepping algorithm for the solution of (3.1) computes $z_{n+1} \simeq z(t+\Delta t)$, given $z_{n}$ and $z_{n-1}$, as follows.

Algorithm 4.1.

1. Set $\tilde{z}_{0}=z_{n}, w_{1}=A(I-P) z_{n}, w_{2}=A(I-P) A z_{n}, u_{1}=A P z_{n}$, and $u_{2}=A P A z_{n}$.

2. Compute $\tilde{z}_{1 / p}=\tilde{z}_{0}-\frac{1}{2}\left(\frac{\Delta t}{p}\right)^{2}\left(w_{1}+u_{1}\right)+\frac{1}{24}\left(\frac{\Delta t}{p}\right)^{4}\left(w_{2}+u_{2}\right)$.

3 . For $m=1 \ldots p-1$, compute

- $v_{1}=-w_{1}+\frac{1}{2}\left(\frac{m \Delta t}{p}\right)^{2} w_{2}-A P \tilde{z}_{m / p}$

- $v_{2}=w_{2}-A P v_{1}$

- $\tilde{z}_{(m+1) / p}=2 \tilde{z}_{m / p}-\tilde{z}_{(m-1) / p}+\left(\frac{\Delta t}{p}\right)^{2} v_{1}+\frac{1}{12}\left(\frac{\Delta t}{p}\right)^{4} v_{2}$.

4. Compute $z_{n+1}=-z_{n-1}+2 \tilde{z}_{1}$.

Here, steps 1-3 correspond to the numerical solution of (4.4) until $\tau=\Delta t$ with the ME approach using the local time step $\Delta \tau=\Delta t / p$. This algorithm requires two multiplications by $A(I-P)$ in step 1 and $2 p$ further multiplications by $A P$. No multiplication by $A$ is needed to compute $A z_{n}$ in step 1 , since

$$
A z_{n}=A(I-P) z_{n}+A P z_{n}=w_{1}+u_{1} .
$$

For $P=0$, that is without any local time stepping, this algorithm reduces to the ME scheme (4.1) above.

To establish the accuracy and stability of the above local time-stepping scheme we shall now show how to rewrite it in "leap-frog manner." To do so, we first need the following technical result.

Lemma 4.2. For $m \geq 2, \tilde{z}_{m / p}$ defined by Algorithm 4.1 satisfies

$$
\begin{aligned}
\tilde{z}_{m / p}= & z_{n}-\frac{m^{2}}{2}\left(\frac{\Delta t}{p}\right)^{2} A z_{n}+\frac{m^{4}}{24}\left(\frac{\Delta t}{p}\right)^{4} A^{2} z_{n} \\
& +\sum_{j=1}^{2(m-1)}\left(\frac{\Delta t}{p}\right)^{2(j+2)} \beta_{j}^{m}(A P)^{j} A A z_{n},
\end{aligned}
$$

where $\beta_{j}^{m}$ are constant. 
Proof. The proof is by induction over $m$.

We first show that (4.6) holds for $m=2$. Starting from the definitions of $w_{1}$ and $w_{2}$ in step 1 of Algorithm 4.1, we find that

$$
\begin{aligned}
& v_{1}=-A(I-P) z_{n}+\frac{1}{2}\left(\frac{\Delta t}{p}\right)^{2} A(I-P) A z_{n}-A P \tilde{z}_{1 / p} \\
& v_{2}=A(I-P) A z_{n}-A P v_{1}
\end{aligned}
$$

Since

$$
\tilde{z}_{1 / p}=z_{n}-\frac{1}{2}\left(\frac{\Delta t}{p}\right)^{2} A z_{n}+\frac{1}{24}\left(\frac{\Delta t}{p}\right)^{4} A^{2} z_{n}
$$

the two expressions for $v_{1}$ and $v_{2}$ simplify as

$$
\begin{aligned}
& v_{1}=-A z_{n}+\frac{1}{2}\left(\frac{\Delta t}{p}\right)^{2} A^{2} z_{n}-\frac{1}{24}\left(\frac{\Delta t}{p}\right)^{4} A P A^{2} z_{n} \\
& v_{2}=A^{2} z_{n}-\frac{1}{2}\left(\frac{\Delta t}{p}\right)^{2} A P A^{2} z_{n}+\frac{1}{24}\left(\frac{\Delta t}{p}\right)^{4} A P A P A^{2} z_{n}
\end{aligned}
$$

We now replace $v_{1}, v_{2}$ in the expression for $\tilde{z}_{2 / p}$ in step 3 . This yields

$$
\begin{aligned}
\tilde{z}_{2 / p}= & z_{n}-2\left(\frac{\Delta t}{p}\right)^{2} A z_{n}+\frac{2}{3}\left(\frac{\Delta t}{p}\right)^{4} A^{2} z_{n} \\
& -\frac{1}{12}\left(\frac{\Delta t}{p}\right)^{6} A P A^{2} z_{n}+\frac{1}{288}\left(\frac{\Delta t}{p}\right)^{8} A P A P A^{2} z_{n} .
\end{aligned}
$$

Hence, $\beta_{1}^{2}=-1 / 12$ and $\beta_{1}^{2}=1 / 288$, which completes the proof for $m=2$.

For higher $m \geq 3$ (and $p \geq 3$ ), the proof is by induction on $m$; those straightforward but tedious calculations are omitted here.

As a consequence, we can rewrite the above local time-stepping algorithm in "leap-frog manner."

Proposition 4.3. The local time-stepping Algorithm 4.1 is equivalent to

$$
z_{n+1}=2 z_{n}-z_{n-1}-\Delta t^{2} A_{p} z_{n},
$$

where $A_{p}$ is defined by

$$
A_{p}=A-\frac{\Delta t^{2}}{12} A^{2}-\frac{2}{p^{2}} \sum_{j=1}^{2(p-1)}\left(\frac{\Delta t}{p}\right)^{2(j+1)} \beta_{j}^{p}(A P)^{j} A^{2} .
$$

This scheme is fourth-order accurate. Furthermore, the matrix $A A_{p}$ is symmetric.

Proof. Recall that $z_{n+1}=-z_{n-1}+2 \tilde{z}_{1}$. We now use (4.6) with $m=p$ to replace $\tilde{z}_{1}$. This yields

$$
z_{n+1}=-z_{n-1}+2 z_{n}-\Delta t^{2} A z_{n}+\frac{\Delta t^{4}}{12} A^{2} z_{n}+\frac{2 \Delta t^{2}}{p^{2}} \sum_{j=1}^{2(p-1)}\left(\frac{\Delta t}{p}\right)^{2(j+1)} \beta_{j}^{p}(A P)^{j} A A z_{n},
$$

which corresponds to (4.11) with $A_{p}$ as in (4.12). 
To prove consistency, we rewrite the scheme as

$$
\frac{z_{n+1}-z_{n-1}+2 z_{n}}{\Delta t^{2}}-\frac{\Delta t^{2}}{12} A^{2} z_{n}-\frac{2}{p^{2}} \sum_{j=1}^{2(p-1)}\left(\frac{\Delta t}{p}\right)^{2(j+1)} \beta_{j}^{p}(A P)^{j} A A z_{n}=-A z_{n} .
$$

From

$$
\frac{z(t+\Delta t)-2 z(t)+z(t-\Delta t)}{\Delta t^{2}}-\frac{\Delta t^{2}}{12} A^{2} z(t)=z^{\prime \prime}(t)+O\left(\Delta t^{4}\right)
$$

and

$$
\sum_{j=1}^{2(p-1)}\left(\frac{\Delta t}{p}\right)^{2(j+1)} \beta_{j}^{p}(A P)^{j} A^{2} z(t)=O\left(\Delta t^{4}\right)
$$

we conclude that Algorithm 4.1 is, indeed, a fourth-order approximation of (3.3).

As the terms $(A P)^{j} A^{2}$ are obviously not symmetric, the matrix $A_{p}$ is not symmetric either. However, since

$$
\left(A(A P)^{j} A\right)^{\top}=A(P A)^{j} A=(A P)^{j} A, \quad j \geq 1,
$$

the matrix $A A_{p}$, indeed, is symmetric.

4.2. Energy conservation and stability. We shall now exhibit a discrete energy which is conserved by the fourth-order local time-stepping scheme (Algorithm 4.1) and, thereby, determine a necessary and sufficient condition for stability. Next, we perform a systematic numerical study to demonstrate the improvement in the CFL condition achieved by slightly extending the overlap of the fine into the coarse region.

First, using Proposition 4.3 we rewrite the local time-stepping scheme as (4.11)(4.12). As a consequence, we can prove the conservation of the following energy.

Proposition 4.4. The fourth-order local time-stepping scheme (Algorithm (4.1)) conserves the following energy:

$$
\begin{aligned}
E^{n+\frac{1}{2}}= & \frac{1}{2}\left[\left\langle\left(A-\frac{\Delta t^{2}}{4} A A_{p}\right) \frac{z_{n+1}-z_{n}}{\Delta t}, \frac{z_{n+1}-z_{n}}{\Delta t}\right\rangle\right. \\
& \left.+\left\langle A A_{p} \frac{z_{n+1}+z_{n}}{2}, \frac{z_{n+1}+z_{n}}{2}\right\rangle\right] .
\end{aligned}
$$

Proof. As $A_{p}$ is not symmetric, we first have to premultiply (4.11) by $A$. Next, we take the inner product of the resulting expression with $z_{n+1}-z_{n-1}$, which yields the conservation of

$$
\left\langle A \frac{z_{n+1}-z_{n}}{\Delta t}, \frac{z_{n+1}-z_{n}}{\Delta t}\right\rangle+\left\langle A A_{p} z_{n+1}, z_{n}\right\rangle
$$

That expression is equal to $E^{n+\frac{1}{2}}$ above, since

$$
\begin{aligned}
\left\langle A A_{p} z_{n+1}, z_{n}\right\rangle= & \left\langle A A_{p} \frac{z_{n+1}+z_{n}}{2}, \frac{z_{n+1}+z_{n}}{2}\right\rangle \\
& -\frac{\Delta t^{2}}{4}\left\langle A A_{p} \frac{z_{n+1}-z_{n}}{\Delta t}, \frac{z_{n+1}-z_{n}}{\Delta t}\right\rangle .
\end{aligned}
$$


TABLE 4.1

$I P-D G \mathcal{P}^{3}$-elements. The smallest eigenvalue of $A A_{p}$ and $A-\left(\Delta t^{2} / 4\right) A A_{p}$ at $\Delta t=\Delta t_{M E}$ with $p=2$ : without overlap (left); with overlap by one element (right).

\begin{tabular}{|c|c|c|}
\hline$h_{\text {coarse }}$ & $A A_{p}$ & $A-\left(\Delta t^{2} / 4\right) A_{p}$ \\
\hline 0.5 & $-5.10^{5}$ & 1.1 \\
\hline 0.2 & $-2.10^{7}$ & 1.1 \\
\hline 0.1 & $-3.10^{8}$ & 1.1 \\
\hline 0.05 & $-5.10^{9}$ & 1.1 \\
\hline 0.025 & $-7.10^{10}$ & 1.1 \\
\hline
\end{tabular}

\begin{tabular}{|c|c|c|}
\hline$h_{\text {coarse }}$ & $A A_{p}$ & $A-\left(\Delta t^{2} / 4\right) A A_{p}$ \\
\hline 0.5 & 1.2 & 1.1 \\
\hline 0.2 & 1.2 & 1.1 \\
\hline 0.1 & 1.2 & 1.1 \\
\hline 0.05 & 1.2 & 1.1 \\
\hline 0.025 & 1.2 & 1.1 \\
\hline
\end{tabular}

TABLE 4.2

$\mathcal{P}^{3}$-FEMs. The smallest eigenvalue of $A A_{p}$ and $A-\left(\Delta t^{2} / 4\right) A A_{p}$ for $\Delta t=\Delta t_{M E}$ with $p=2$ without overlap.

\begin{tabular}{|c|c|c|}
\hline$h_{\text {coarse }}$ & $A A_{p}$ & $A-\left(\Delta t^{2} / 4\right) A A_{p}$ \\
\hline 0.5 & 1.2 & 0.15 \\
\hline 0.2 & 1.2 & 0.9 \\
\hline 0.1 & 1.2 & 0.53 \\
\hline 0.05 & 1.2 & 1.1 \\
\hline 0.025 & 1.2 & 1.1 \\
\hline
\end{tabular}

Since both $A$ and $A A_{p}$ are symmetric matrices, $E^{n+\frac{1}{2}}$ is a symmetric quadratic form which is positive for $\Delta t$ sufficiently small. Hence, the local time-stepping scheme will be stable if and only if the two matrices $\left(A-\frac{\Delta t^{2}}{4} A A_{p}\right)$ and $A A_{p}$ are positive semidefinite. For $p=1$, we have $A A_{p}=A\left(A-\frac{\Delta t}{12} A^{2}\right)$ and, thus, we recover the CFL condition of the ME scheme:

$$
\Delta t \leq \frac{2 \sqrt{3}}{\sqrt{\lambda_{\max }}}=\sqrt{3} \Delta t_{L F}=\Delta t_{M E},
$$

where $\lambda_{\max }$ is the largest eigenvalue of $A$.

For $p \geq 2$, the CFL condition cannot be determined analytically. Thus, we shall again provide a systematic numerical study to evaluate the stability of Algorithm 4.1 in a typical situation.

We consider the same one-dimensional problem as in section 3.3 but now use an IP-DG discretization with $\mathcal{P}^{3}$-elements with (small) penalization $\alpha=7$ to achieve fourth-order accuracy both in space and time. In the absence of local time stepping, that is with $p=1$, the maximal time step dictated by the equidistant (coarse) mesh is $\Delta t_{M E}=0.265 h_{\text {coarse }}$. For $p \geq 2$, we choose the same optimal time step $\Delta t=\Delta t_{M E}$ and compute the smallest eigenvalue of $\left(A-\frac{\Delta t^{2}}{4} A A_{p}\right)$ and $A A_{p}$, respectively - both need to be positive for stability. From Table 4.1 we conclude that Algorithm 4.1 without overlap is unstable for the maximal time step. However, as we extend by a single adjacent element the region where smaller time steps are used, the fourth-order local time-stepping scheme becomes stable independently of $p$ and $h_{\text {coarse }}$.

Next, we consider continuous piecewise $\mathcal{P}^{3}$ finite elements with mass lumping. Since the CFL condition of the leap-frog scheme is $\Delta t_{L F}=0.232 h_{\text {coarse }}$ [13], the corresponding CFL condition of the ME scheme is $\Delta t_{M E}=\sqrt{3} \Delta t_{L F}=0.401 h_{\text {coarse }}$. Again, we automatically include those degrees of freedom that belong to both a fine and a coarse cell into $z^{[\mathrm{fine}]}$. Remarkably, no overlap is needed here for the local time-stepping scheme to remain stable, regardless of $h_{\text {coarse }}$, as shown in Table 4.2 for $p=2$. 
4.3. Local time stepping of arbitrary order. The ME approach used in section 4.1 to extend the local time-stepping approach to fourth order can be generalized to arbitrary accuracy. Since the procedure parallels that used in section 4.1, we shall omit details and state only key results.

Starting again from the Taylor expansion

$$
z^{[\mathrm{coarse}]}(t+\theta \Delta t)=z^{[\mathrm{coarse}]}(t)+\sum_{i=1}^{2 s-2} \frac{\theta^{i} \Delta t^{i}}{i !} \frac{d^{i} z^{[\mathrm{coarse}]}}{d \theta^{i}}(t)+O\left(\Delta t^{2 s-1}\right),
$$

we use the fact that

$$
\frac{d^{2 i} z^{[\mathrm{coarse}]}}{d t^{2 i}}(t)=(I-P) \frac{d^{2 i} z}{d t^{2 i}}(t)=(I-P)(-A)^{i} z(t)
$$

to obtain

$$
\begin{aligned}
z^{[\text {coarse }]}(t+\theta \Delta t)= & z^{[\text {coarse }]}(t)+\sum_{i=1}^{s-1} \frac{\theta^{2 i} \Delta t^{2 i}}{(2 i) !}(I-P)(-A)^{i} z(t) \\
& +\sum_{i=1}^{s-1} \frac{\theta^{2 i-1} \Delta t^{2 i-1}}{(2 i-1) !} \frac{d^{2 i-1} z^{[\text {coarse }]}}{d \theta^{2 i-1}}(t)+O\left(\Delta t^{2 s-1}\right) .
\end{aligned}
$$

Next, we use (4.15) in (3.7) to replace $z^{[\text {coarse] }}$, which yields

$$
\begin{aligned}
z(t+\Delta t)-2 z(t)+z(t-\Delta t) \simeq & -\Delta t^{2} A(I-P) z(t)+2 \sum_{i=1}^{s-1} \frac{\Delta t^{2 i+2}}{(2 i+2) !} A(I-P)(-A)^{i} z(t) \\
& -\Delta t^{2} \int_{-1}^{1}(1-|\theta|) A z^{[\mathrm{fine}]}(t+\theta \Delta t) d \theta
\end{aligned}
$$

Similar to section 3.1, we now approximate the right-hand side of (4.16) by solving

$$
\left\{\begin{array}{l}
\frac{d^{2} \tilde{z}}{d \tau^{2}}=-A(I-P) z(t)-\sum_{i=1}^{s} \frac{\tau^{2 i}}{2 i !} A(I-P)(-A)^{i} z(t)-A P \tilde{z}(\tau), \\
\tilde{z}(0)=z(t), \quad \frac{d \tilde{z}}{d t}(0)=0
\end{array}\right.
$$

and then, again, use

$$
z(t+\Delta t)+z(t-\Delta t) \simeq 2 \tilde{z}(\Delta t) .
$$

Thus, we approximate the right side of (4.16) by solving (4.17) for $0 \leq \tau \leq \Delta t$ and then use (4.18) to compute $z(t+\Delta t)$. The last term on the right side of (4.17) explicitly depends on $\tilde{z}(\tau)$, but it involves only the unknowns associated with the nonzero entries in $P$. If those nonzero entries occupy only a small fraction of all unknowns, the additional effort from solving (4.17) will be small. Clearly, in doing so we must ensure that the overall numerical scheme remains $2 s$ th-order accurate in time.

In summary, the local time-stepping algorithm of order $2 s$ for the solution of (3.1) computes $z_{n+1} \simeq z(t+\Delta t)$, given $z_{n}$ and $z_{n-1}$, as follows. 
Algorithm 4.5.

1. Set $\tilde{z}_{0}=z_{n}, \quad w_{m}=A(I-P) A^{m-1} z_{n}, \quad u_{m}=A P A^{m-1} z_{n}, \quad m=1, \ldots, s$.

2. Compute

- $v_{m}=(-1)^{m}\left(w_{m}+u_{m} \tilde{z}_{0}\right), \quad m=1, \ldots, s$;

- $\tilde{z}_{1 / p}=\tilde{z}_{0}+\sum_{m=1}^{s} \frac{1}{(2 m) !}\left(\frac{\Delta t}{p}\right)^{2 m} v_{m}$.

3. For $m=1, \ldots, p-1$, compute

- $v_{1}=-\left(w_{1}+\sum_{l=1}^{s} \frac{(-1)^{l}}{(2 l) !} w_{l}\right)-A P \tilde{z}_{m / p}, \quad k=1, \ldots, s ;$

- $v_{k}=(-1)^{k}\left(w_{k}+\sum_{l=k+1}^{s} \frac{(-1)^{l-k}}{(2(l-k)) !} w_{l}\right)-A P v_{k-1}, \quad k=2, \ldots, s$;

- $\tilde{z}_{(m+1) / p}=2 \tilde{z}_{m / p}-\tilde{z}_{(m-1) / p}+\sum_{k=1}^{s} \frac{1}{(2 m) !}\left(\frac{\Delta t}{p}\right)^{2 k} a_{k}$.

4. Compute $z_{n+1}=-z_{n-1}+2 \tilde{z}_{1}$.

Steps 1-3 compute the numerical solution of (4.17) at time $\tau=\Delta t$ using the 2sthorder ME scheme with local time step $\Delta \tau=\Delta t / p$. Note that we recover the standard ME scheme by setting $P=0$. Clearly, this algorithm only requires $s$ multiplications by $A(I-P)$ and $p s$ multiplications by $A P$. No multiplication by $A$ is needed to compute $A^{m-1} z_{n}$ in step 1 , since

$$
A^{m-1} z_{n}=A(I-P) A^{m-1} z_{n}+A P A^{m-1} z_{n}=w_{m}+u_{m} .
$$

We now rewrite the above alogrithm in "leap-frog manner" to determine the accuracy and establish the stability of the above algorithm.

Proposition 4.6. The local time-stepping method (Algorithm 4.5) of order $2 s$ is equivalent to

$$
z_{n+1}=2 z_{n}-z_{n-1}-\Delta t^{2} A_{p} z_{n}
$$

where $A_{p}$ is defined by

$$
A_{p}=A-2 \sum_{j=2}^{s} \frac{\Delta t^{2(j-1)}}{(2 j) !} A^{j}-\frac{2}{p^{2}} \sum_{j=1}^{(p-1) s}\left(\frac{\Delta t}{p}\right)^{2(s-1+j)} \gamma_{j}^{p}(A P)^{j} A^{s} .
$$

This scheme is 2 sth-order accurate. Furthermore, the matrix $A^{s-1} A_{p}$ is symmetric.

Proof. We do not detail the proof of the first part of the proposition, which is similar to that of Propositions 3.3 and 4.3. To prove consistency, we rewrite the scheme as

$$
\frac{z_{n+1}-2 z_{n}-z_{n-1}}{\Delta t^{2}}-2 \sum_{j=2}^{s} \frac{\Delta t^{2(j-1)}}{(2 j) !} A^{j}-\frac{2}{p^{2}} \sum_{j=1}^{(p-1) s}\left(\frac{\Delta t}{p}\right)^{2(s-1+j)} \gamma_{j}^{p}(A P)^{j} A^{s}=-A z_{n} .
$$

Since

$$
\frac{z(t+\Delta t)-2 z(t)+z(t-\Delta t)}{\Delta t^{2}}-2 \sum_{j=2}^{s} \frac{\Delta t^{2(j-1)}}{(2 j) !} A^{j}=z^{\prime \prime}(t)+O\left(\Delta t^{2 s}\right)
$$

and

$$
\sum_{j=1}^{(p-1) s}\left(\frac{\Delta t}{p}\right)^{2(s-1+j)} \gamma_{j}^{p}(A P)^{j} A^{s}=O\left(\Delta t^{2 s}\right),
$$

Algorithm 4.5 is a 2 sth-order approximation of (3.3). 
As the matrices $(A P)^{j} A^{s}$ are generally not symmetric, the matrix $A_{p}$ is not symmetric either. However, since

$$
\left(A^{s-1}(A P)^{j} A^{s}\right)^{\top}=A^{s}(P A)^{j} A^{s-1}=A^{s-1}(A P)^{j} A^{s}, \quad j \geq 1,
$$

the matrix $A^{s-1} A_{p}$ is, indeed, symmetric.

We can now prove the conservation of a discrete energy.

Proposition 4.7. The local time-stepping scheme (Algorithm 4.5) of order $2 \mathrm{~s}$ conserves the following energy:

$$
\begin{aligned}
E^{n+\frac{1}{2}}= & \frac{1}{2}\left[\left\langle\left(A^{s-1}-\frac{\Delta t^{2}}{4} A^{s-1} A_{p}\right) \frac{z_{n+1}-z_{n}}{\Delta t}, \frac{z_{n+1}-z_{n}}{\Delta t}\right\rangle\right. \\
& \left.+\left\langle A^{s-1} A_{p} \frac{z_{n+1}+z_{n}}{2}, \frac{z_{n+1}+z_{n}}{2}\right\rangle\right] .
\end{aligned}
$$

Proof. As $A_{p}$ is not symmetric, we first premultiply (4.19) by $A^{s-1}$. Next, we take the inner product of the resulting expression by $z_{n+1}-z_{n-1}$, which yields the conservation of

$$
\left\langle A^{s-1} \frac{z_{n+1}-z_{n}}{\Delta t}, \frac{z_{n+1}-z_{n}}{\Delta t}\right\rangle+\left\langle A^{s-1} A_{p} z_{n+1}, z_{n}\right\rangle .
$$

That expression is equal to $E^{n+\frac{1}{2}}$ above, since

$$
\begin{aligned}
\left\langle A^{s-1} A_{p} z_{n+1}, z_{n}\right\rangle= & \left\langle A^{s-1} A_{p} \frac{z_{n+1}+z_{n}}{2}, \frac{z_{n+1}+z_{n}}{2}\right\rangle \\
& -\frac{\Delta t^{2}}{4}\left\langle A^{s-1} A_{p} \frac{z_{n+1}-z_{n}}{\Delta t}, \frac{z_{n+1}-z_{n}}{\Delta t}\right\rangle .
\end{aligned}
$$

Since both $A^{s-1}$ and $A^{s-1} A_{p}$ are symmetric matrices, $E^{n+\frac{1}{2}}$ is a symmetric quadratic form which is positive for $\Delta t$ sufficiently small. Hence, the local timestepping scheme will be stable if and only if the two matrices $\left(A^{s-1}-\frac{\Delta t^{2}}{4} A^{s-1} A_{p}\right)$ and $A^{s-1} A_{p}$ are positive definite.

5. Numerical results. We shall now present numerical experiments that confirm the expected order of convergence and demonstrate the versatility of the above local time-stepping methods. First, we consider a simple one-dimensional test problem to show that the different local time-stepping schemes presented above, indeed, yield the expected overall rate of convergence when combined with a spatial finite element discretization of comparable accuracy, independently of the number of local time steps $p$. Then, we consider wave propagation in two space dimensions with a locally highly refined mesh to illustrate the usefulness of local time stepping in the presence of complex geometry.

5.1. Convergence study. We consider the one-dimensional wave equation with constant wave speed $c=1$ on the interval $\Omega=[0 ; 6]$ with periodic boundary conditions. The initial conditions are chosen to yield the exact solution $u_{e x}(x, t)=$ $\sin (8 \pi(x-t) / 3)$, which corresponds to a sinusoidal wave propagating to the right. Again, we divide $\Omega$ into three equal parts. The left and right intervals, $[0 ; 2]$ and $[4 ; 6]$, respectively, are discretized with an equidistant mesh of size $h_{\text {coarse }}$, whereas the interval $[2 ; 4]$ is discretized with an equidistant mesh of size $h_{\text {fine }}=h_{\text {coarse }} / p$. 

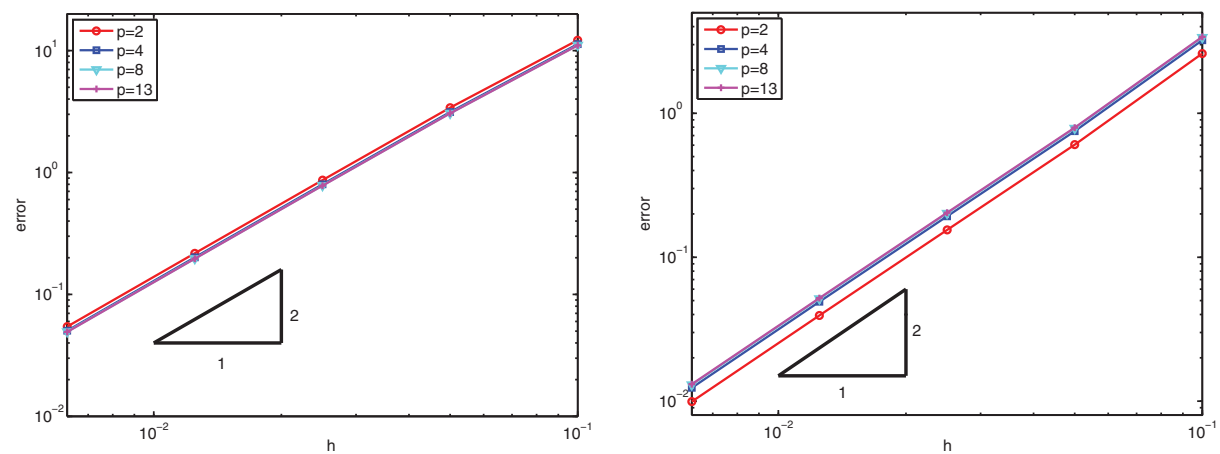

FIG. 5.1. Error vs. $h=h_{\text {coarse for }} P^{1}$ finite elements with $p=2,4,8,13: I P-D G$ (left) and continuous FE (right).

Hence, the two outer intervals correspond to the coarse region and the inner interval $[2 ; 4]$ to the refined region-refer to section 3.3 for further details.

For every time step $\Delta t$, we shall take $p \geq 2$ local steps of size $\Delta \tau=\Delta t / p$ in the refined region, with the second-order local time-stepping Algorithm 3.1. We choose an overlap of two and set in all instances $\Delta t=\Delta t_{L F}$, the largest time step allowed by the leap-frog method on an equidistant mesh with mesh size $h=h_{\text {coarse }}$.

First, we consider an IP-DG discretization with $\mathcal{P}^{1}$-elements and (small) penalty parameter $\alpha=2$, as described in section 3.3, and the sequence of meshes $\mathcal{T}_{h}, h_{\text {coarse }}=$ $0.1,0.05,0.025,0.0125,0.00625$. As we systematically reduce the global mesh size $h_{\text {coarse, }}$, while simultaneously reducing $\Delta t$, we monitor the $L^{2}$ space-time error in the numerical solution $\left\|u-u_{e x}\right\|_{L^{2}\left(0, T ; L^{2}(\Omega)\right)}$ until the final time $T=60$. In the left frame in Figure 5.1, the numerical error is shown vs. the mesh size $h=h_{\text {coarse }}$. Regardless of the number of local time steps $p=2,4,8,13$, the numerical method converges with order two.

We now repeat the same experiment with $P^{1}$ continuous finite elements with mass lumping for the same sequence of meshes. As shown in the right frame of Figure 5.1, the local time-stepping method again yields overall second-order convergence independently of $p$.

Next, we consider the fourth-order time-stepping scheme (Algorithm 4.1) and combine it with either a continuous FE or the IP-DG discretization with $\mathcal{P}^{3}$-elements. Thus, we expect both numerical schemes to exhibit overall fourth-order convergence with respect to the $L^{2}$-norm. Again, we choose an overlap of two and let $\Delta t=\Delta t_{M E}$, the largest possible time step allowed by the ME approach on an equidistant mesh with $h=h_{\text {coarse }}$. In Figure 5.2 we display the space-time $L^{2}$-errors of the numerical solutions for the sequence of meshes $h_{\text {coarse }}=0.2,0.1,0.05,0.025,0.0125$ and different values of $p$. Both the continuous FEM with mass lumping and the IP-DG method, here with $\alpha=7$, yield the expected fourth-order convergence.

Finally, to validate the order of convergence of the sixth-order time-stepping scheme (Algorithm 4.5 with $s=3$ ), we consider the IP-DG method with $\mathcal{P}^{5}$-elements, where we set $\alpha=16$. As above we choose an overlap of two elements and set the time step to its (maximal) optimal value $\Delta t=\Delta t_{M E}$, the largest possible time step allowed by the ME approach of order six on an equidistant mesh with $h=h_{\text {coarse }}$. Again, the numerical results shown in Figure 5.3 for $p=2$ corroborate the expected sixth order of convergence. 

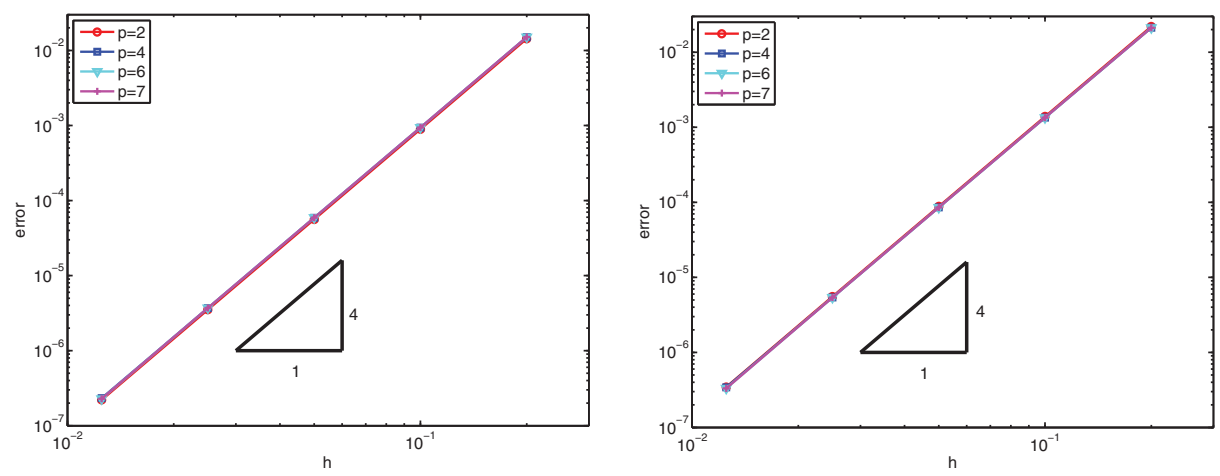

Fig. 5.2. Error vs. $h=h_{\text {coarse for }} P^{3}$ finite elements with $p=2,4,6,7:$ IP-DG (left) and continuous FE (right).

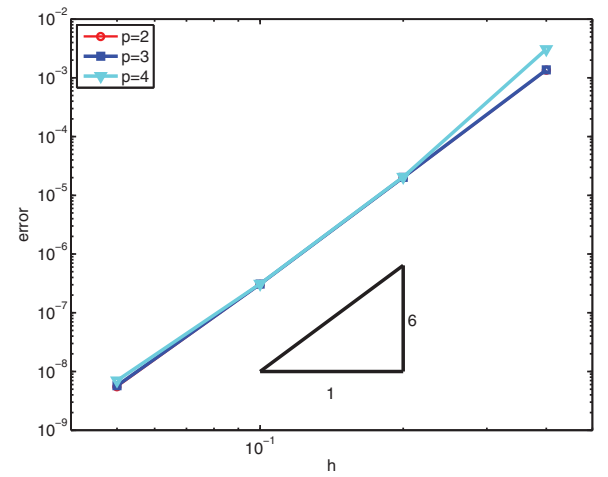

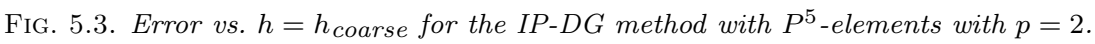

5.2. Two-dimensional example. To illustrate the usefulness of the local timestepping methods presented above, we consider the wave equation (2.1)-(2.4) with constant speed $c=1$ in a computational domain $\Omega$, that consists of two $1 \times 0.95$ rectangles connected by a very narrow $0.004 \times 0.1$ channel - see Figure 5.4. We impose homogeneous Neumann conditions on the boundary of $\Omega$ and choose as initial conditions

$$
\begin{aligned}
& u_{0}(x)=\left\{\begin{array}{lr}
\exp \left(\left\|x-x_{0}\right\|^{2} / r^{2}\right), & \left\|x-x_{0}\right\| \leq \sqrt{2} r, \\
0, & \text { otherwise },
\end{array}\right. \\
& v_{0}(x)=0,
\end{aligned}
$$

where $x_{0}=(0,0.25)$ and $r=0.025$.

For the spatial discretization we opt for the IP-DG method with $P^{3}$ triangular elements and $\alpha=11$. Hence, to resolve the propagating wave with sufficient accuracy, we find that a mesh size $h_{\text {coarse }}=0.0125$ is approximately needed. However, such triangles do not even fit inside the narrow gap, which requires $h_{\text {fine }} \simeq h_{\text {coarse }} / 16.4$ to resolve its geometric features, as shown in Figure 5.5.

For the time discretization we choose the fourth-order local time-stepping method from section 4.1. Thus, the numerical method is fourth-order accurate in both space and time under the CFL restriction $\Delta t \leq 0.14 h$, determined experimentally. If the same (global) time step $\Delta t$ were used everywhere in $\Omega$, it would need to be about 


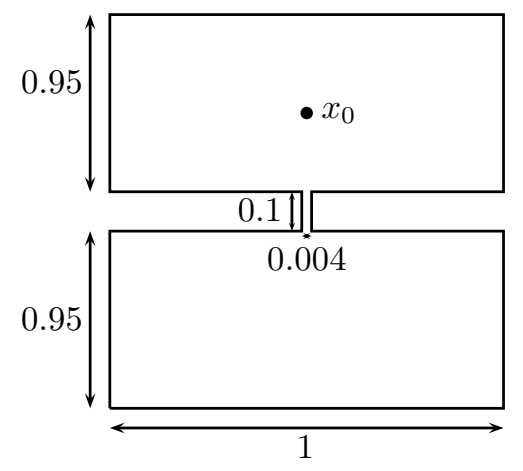

FIG. 5.4. Two-dimensional example: the computational domain $\Omega$.
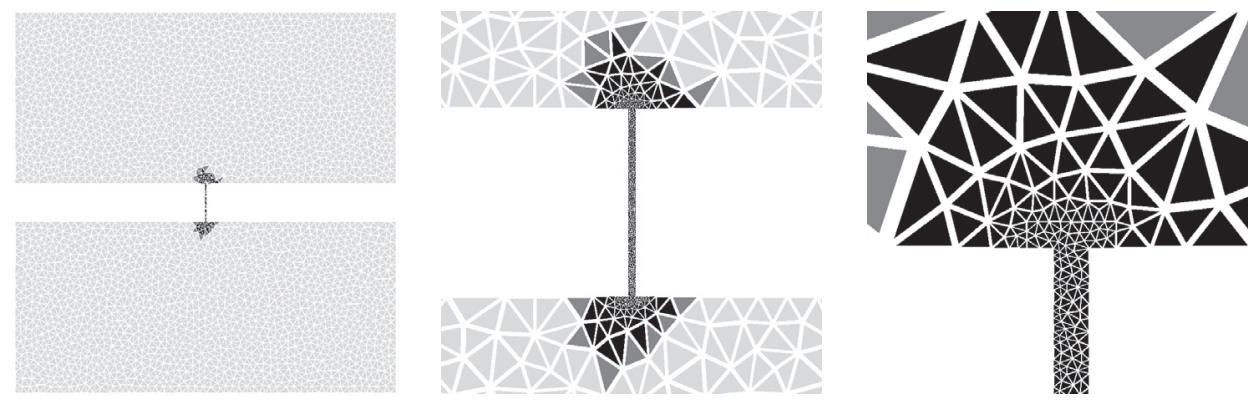

FIG. 5.5. The triangular mesh at various magnification rates: the black and dark gray triangles belong to the "fine" mesh and the light gray triangles to the "coarse" mesh.

seventeen times smaller than necessary in most of $\Omega$ for stability reasons only. Instead, we shall use the fourth-order local time stepping with $p=17$, which for every time step $\Delta t=0.14 h_{\text {coarse }}$ takes seventeen local time steps $\Delta \tau=\Delta t / 17$ inside the highly refined region.

In dimension two or higher, the boundary between the fine and the coarse meshes is typically not as well-defined as in one space dimension while the transition between larger and smaller elements is more gradual. Given $h_{\text {coarse }}$, here the fine mesh corresponds to all triangles with $h<0.95 h_{\text {coarse }}$ in size, that is the black triangles in Figure 5.5. Moreover, to ensure stability with the optimal time step, dictated by $h_{\text {coarse, }}$, we also include those triangles directly adjacent to the fine region; these few additional elements, depicted in dark gray in Figure 5.5, correspond to an overlap as in section 3.3. All remaining (light gray) triangles belong to the coarse mesh. The degrees of freedom corresponding to the fine mesh in the finite element solution are then selected merely by setting the corresponding diagonal entries of the matrix $P$ to one - see section 3.1.

For the first time step, we approximate $U(\Delta t)$ by a fifth-order Taylor expansion, where all higher derivatives are obtained by repeated differentiation of (3.1), that is

$$
U_{1}=U_{0}+\Delta t V_{0}+\frac{\Delta t^{2}}{2} M^{-1} K U_{0}+\frac{\Delta t^{3}}{6} M^{-1} K V_{0}+\frac{\Delta t^{4}}{24}\left(M^{-1} K\right)^{2} U_{0} .
$$

In Figure 5.6 snapshots of the numerical solution $u$ are shown at times $t=0.09$, $0.17,0.35,0.44,0.61,0.7$ - to improve the contrast in the figure we have magnified by 

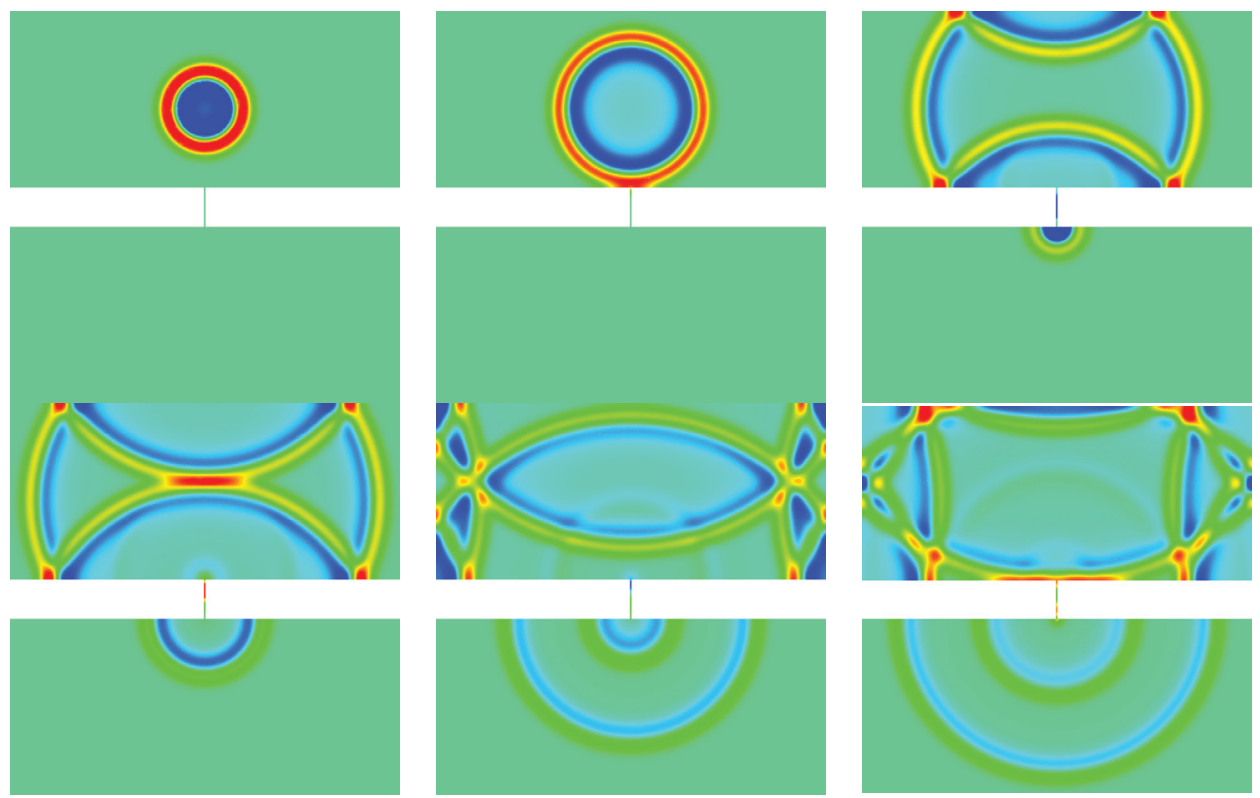

Fig. 5.6. Two-dimensional example: the solution is shown at times $t=0.09,0.17,0.35,0.44$, 0.61, 0.7-the solution is magnified by a factor of five in the lower part.
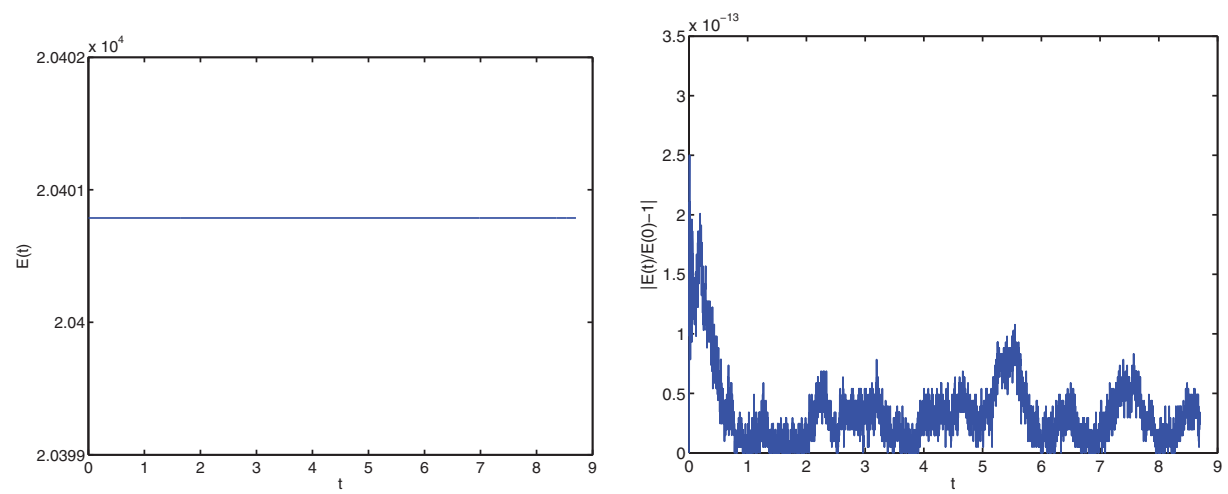

Fig. 5.7. Time evolution of the discrete energy defined in (4.13) (left) and of its relative variation $|E(t) / E(0)-1|$ (right).

a factor of five the solution in the lower part of the computational domain. A circular wave is initiated by the Gaussian pulse centered about $x_{0}$ in the upper region, which propagates outward until it impinges on the lower boundary at $t=0.17$. Then, a fraction of the wave penetrates the channel and generates a circular outgoing wave as it reaches the opposite lower region. Further reflections occur as the wave moves back and forth inside the channel, subsequently generating multiple circular waves in the upper and lower domains.

Finally, we verify that the discrete numerical energy defined in (4.13) is truly conserved over time. In Figure 5.7 we follow the time evolution of the energy and its relative variation, which remains within machine precision for the entire simulation until $T=8.7$, that is during 5000 time steps. 
6. Conclusion. We have presented explicit local time-stepping methods for the wave equation, which allow arbitrarily small time steps precisely where the smallest elements in the mesh are located. When combined with a symmetric finite element discretization in space with an essentially diagonal mass matrix, the resulting discrete time-marching scheme remains truly explicit, while it also conserves a discrete energy. Starting from the standard second-order "leap-frog" scheme, we have derived local time integration methods of arbitrary order. When the "fine" region, where local time steps are used, slightly extends into the surrounding "coarse" region of the mesh, we find that the resulting numerical scheme permits the use of the optimal maximal time step, dictated by the coarse mesh size.

Since the local time-stepping methods presented here are truly explicit, their parallel implementation is straightforward. Let $\Delta t$ denote the time step imposed by the CFL condition in the coarser part of the mesh. Then, during every (global) time step $\Delta t$, each local time step of size $\Delta t / p$ inside the fine region of the mesh, with $p \geq 2$ any integer, simply corresponds to sparse matrix-vector multiplications that involve only the degrees of freedom associated with the fine region of the mesh. Those "fine" degrees of freedom can be selected individually and without any restriction by setting the corresponding entries in the diagonal projection matrix $P$ to one; in particular, no adjacency or coherence in the numbering of the degrees of freedom is assumed. Hence, the implementation is straightforward and requires no special data structures.

The local time-stepping methods derived here for the scalar wave equation immediately apply to more general second-order hyperbolic problems, as in elasticity or electromagnetics, for which either symmetric DG [24,25] or mass lumping techniques are available [19]. They also generalize to the situation of nonzero forcing. Clearly these time-stepping schemes can also be combined with finite difference methods on highly stretched grids, such as body-fitted grids [42], if the underlying finite difference discretization leads to a symmetric stiffness matrix. In the presence of hierarchical mesh refinement, each local time step in the fine region can itself include further local time steps inside a smaller subregion with an even higher degree of local mesh refinement.

Acknowledgments. We thank Ernst Hairer and Christian Lubich for useful comments and suggestions.

\section{REFERENCES}

[1] M. Ainsworth, P. Monk, And W. Muniz, Dispersive and dissipative properties of discontinuous Galerkin finite element methods for the second-order wave equation, J. Sci. Comput., 27 (2006), pp. 5-40.

[2] L. Anné, P. Joly, And Q. H. Tran, Construction and analysis of higher order finite difference schemes for the $1 D$ wave equation, Comput. Geosci., 4 (2000), pp. 207-249.

[3] D. N. Arnold, F. Brezzi, B. Cockburn, and L. D. Marini, Unified analysis of discontinuous Galerkin methods for elliptic problems, SIAM J. Numer. Anal., 39 (2002), pp. 1749-1779.

[4] G. A. BAKER, Error estimates for finite element methods for second order hyperbolic equations, SIAM J. Numer. Anal., 13 (1976), pp. 564-576.

[5] G. A. Baker And V. A. Dougalis, The effect of quadrature errors on finite element approximations for second order hyperbolic equations, SIAM J. Numer. Anal., 13 (1976), pp. 577-598.

[6] E. BÉCAChe, P. Joly, And J. RodríGuez, Space-time mesh refinement for elastodynamics. Numerical results, Comput. Methods Appl. Mech. Engrg., 194 (2005), pp. 355-366.

[7] R. Carpentier, A. De La Bourdonnaye, and B. Larrouturou, On the derivation of the modified equation for the analysis of linear numerical methods, RAIRO Modél. Math. Anal. Numér., 31 (1997), pp. 459-470. 
[8] P. Ciarlet, The Finite Element Method for Elliptic Problems, North-Holland, Amsterdam, 1978.

[9] B. CockBurn, Discontinuous Galerkin methods for convection-dominated problems, in HighOrder Methods for Computational Physics, Lect. Notes Comput. Sci. Eng. 9, T. Barth and H. Deconink, eds., Springer-Verlag, Berlin, 1999, pp. 69-224.

[10] B. Cockburn, G. Karniadakis, and C.-W. Shu, The development of discontinuous Galerkin methods, in Discontinuous Galerkin Methods: Theory, Computation and Applications, Lect. Notes Comput. Sci. Eng. 11, B. Cockburn, G. Karniadakis, and C.-W. Shu, eds., Springer-Verlag, Berlin, 2000, pp. 3-50.

[11] B. CockBuRn AND C.-W. Shu, Runge-Kutta discontinuous Galerkin methods for convectiondominated problems, J. Sci. Comput., 16 (2001), pp. 173-261.

[12] G. C. Cohen, Higher-Order Numerical Methods for Transient Wave Equations, Sci. Comput., Springer-Verlag, Berlin, 2002.

[13] G. Cohen and S. Fauqueux, Mixed spectral finite elements for the linear elasticity system in unbounded domains, SIAM J. Sci. Comput., 26 (2005), pp. 864-884.

[14] G. Cohen, P. Joly, J. E. Roberts, and N. Tordjman, Higher order triangular finite elements with mass lumping for the wave equation, SIAM J. Numer. Anal., 38 (2001), pp. 2047-2078.

[15] F. Collino, T. Fouquet, And P. Joly, A conservative space-time mesh refinement method for the 1-D wave equation. I. Construction, Numer. Math., 95 (2003), pp. 197-221.

[16] F. Collino, T. Fouquet, And P. Joly, A conservative space-time mesh refinement method for the 1-D wave equation. II. Analysis, Numer. Math., 95 (2003), pp. 223-251.

[17] F. Collino, T. Fouquet, And P. Joly, Conservative space-time mesh refinement methods for the FDTD solution of Maxwell's equations, J. Comput. Phys., 211 (2006), pp. 9-35.

[18] M. A. Dablain, High order differencing for the scalar wave equation, in SEG Technical Program Expanded Abstracts, Vol. 3, Society of Exploration Geophysicists, Tulsa, OK, 1984, pp. 854-854.

[19] A. Elmkies And P. Joly, Finite elements and mass lumping for Maxwell's equations: The $2 D$ case, C. R. Acad. Sci. Paris Sér. I, 324 (1997), pp. 1287-1293.

[20] M. J. Gander, L. Halpern, And F. NAtAF, Optimal Schwarz waveform relaxation for the one dimensional wave equation, SIAM J. Numer. Anal., 41 (2003), pp. 1643-1681.

[21] J. Gilbert AND P. Joly, Higher order time stepping for second order hyperbolic problems and optimal CFL conditions, in Partial Differential Equations, Comput. Methods Appl. Sci. 16, Springer-Verlag, Dordrecht, The Netherlands, 2006.

[22] F. X. Giraldo AND M. A. TAYLOR, A diagonal-mass-matrix triangular-spectral-element method based on cubature points, J. Engrg. Math., 56 (2006), pp. 307-322.

[23] M. J. Grote, A. Schneebeli, and D. Schötzau, Discontinuous Galerkin finite element method for the wave equation, SIAM J. Numer. Anal., 44 (2006), pp. 2408-2431.

[24] M. J. Grote, A. Schneebeli, And D. SchötZau, Interior penalty discontinuous Galerkin method for Maxwell's equations: Energy norm error estimates, J. Comput. Appl. Math., 204 (2007), pp. 375-386.

[25] M. J. Grote, A. Schneebeli, and D. Schötzau, Interior penalty discontinuous Galerkin method for Maxwell's equations: Optimal $L^{2}$-norm error estimates, IMA J. Numer. Anal., 28 (2008), pp. 440-468.

[26] M. J. Grote And D. Schötzau, Optimal error estimates for the fully discrete interior penalty $D G$ method for the wave equation, J. Sci. Comput., to appear.

[27] B. Gustafsson and E. MossberG, Time compact high order difference methods for wave propagation, SIAM J. Sci. Comput., 26 (2004), pp. 259-271.

[28] B. GustafsSOn And P. WAHLund, Time compact high order difference methods for wave propagation, 2D, J. Sci. Comput., 25 (2005), pp. 195-211.

[29] E. Hairer, C. Lubich, and G. Wanner, Geometric Numerical Integration, StructurePreserving Algorithms for Ordinary Differential Equations, Springer Ser. Comput. Math. 31, Springer-Verlag, Berlin, 2006.

[30] L. HALPERN, Local space-time refinement for the one-dimensional wave equation, J. Comput. Acoust., 13 (2005), pp. 547-568.

[31] J. P. Hennart, Topics in finite element discretization of parabolic evolution problems, in Numerical Analysis, Proceedings of the Third IIMAS Workshop Held at Cocoyoc, Mexico, Lecture Notes in Math. 909, J. P. Hennart, ed., Springer-Verlag, New York, 1982, pp. 185199.

[32] T. Hughes, The Finite Element Method: Linear Static and Dynamic Finite Element Analysis, Prentice-Hall, Englewood Cliffs, NJ, 1987.

[33] P. Joly And J. RodríGuez, An error analysis of conservative space-time mesh refinement methods for the one-dimensional wave equation, SIAM J. Numer. Anal., 43 (2005), pp. 825859. 
[34] H.-O. Kreiss, N. A. Petersson, And J. Yström, Difference approximations for the second order wave equation, SIAM J. Numer. Anal., 40 (2002), pp. 1940-1967.

[35] B. Leimkuhler And S. Reich, Simulating Hamiltonian Dynamics, Cambridge Monogr. Appl. Comput. Math., Cambridge University Press, Cambridge, 2004.

[36] J. Lions and E. Magenes, Non-homogeneous Boundary Value Problems and Applications, Vol. I, Springer-Verlag, New York, 1972.

[37] C. LuBICH, private communication, Mathematisches Institut, Univerität Tübingen, Germany, 2006.

[38] Y. Maday And A. Patera, Spectral element methods for the incompressible Navier-Stokes equations, in State-of-the-Art Surveys in Computational Mechanics, A. Noor and J. T. Oden, eds., ASME, New York, 1989, pp. 71-143.

[39] S. PIPERNO, Symplectic local time-stepping in non-dissipative DGTD methods applied to wave propagation problems, Modél. Math. Anal. Numér., 40 (2006), pp. 815-841.

[40] G. Seriani And E. Priolo, Spectral element method for acoustic wave simulation in heterogeneous media, Finite Elem. Anal. Des., 16 (1994), pp. 337-348.

[41] G. R. Shubin And J. B. Bell, A modified equation approach to constructing fourth order methods for acoustic wave propagation, SIAM J. Sci. Statist. Comput., 8 (1987), pp. 135151.

[42] V. Villamizar, Time-dependent numerical method with boundary-conforming curvilinear coordinates applied to wave interactions with prototypical antennas, J. Comput. Phys., 177 (2002), pp. 1-36. 